\title{
Log-linear Dynamics and Local Potential
}

\author{
DaIJIRo OKADA $^{\dagger}$ AND Olivier TercieuX ${ }^{\ddagger}$
}

[This version: November 28, 2008]

\begin{abstract}
We show that local potential maximizer ([15]) with constant weights is stochastically stable in the log-linear dynamics provided that the payoff function or the associated local potential function is supermodular. We illustrate and discuss, through a series of examples, the use of our main results as well as other concepts closely related to local potential maximizer: weighted potential maximizer, pdominance. We also discuss the log-linear processes where each player's stochastic choice rule converges to the best response rule at different rates. For $2 \times 2$ games, we examine a modified log-linear dynamics (relative log-linear dynamics) under which local potential maximizer with strictly positive weights is stochastically stable. This in particular implies that for $2 \times 2$ games a strict $\left(p_{1}, p_{2}\right)$-dominant equilibrium with $p_{1}+p_{2}<1$ is stochastically stable under the new dynamics. (Journal of Economic Literature Classification Number: C72, C73)

Keywords: Log-linear dynamics, Relative log-linear dynamics, Stochastic stability, Local potential maximizer, p-dominant equilibrium, Equilibrium selection, Stochastic order, Comparison of Markov Chains
\end{abstract}

$\dagger$ Department of Economics, Rutgers, The State University of New Jersey, NJ, USA. Email: okada@econ.rutgers.edu

$\ddagger$ Paris School of Economics and CNRS, Paris, France. Email: tercieux@pse.ens.fr 


\section{Acknowledgement}

This research was initiated while the authors were members of School of Social Science at Institute for Advanced Study, Princeton, USA, during 2007-2008 academic year. Okada gratefully acknowledges the support at the institute through Richard B, Fisher membership. Tercieux likewise acknowledges the support through Deutsche Bank membership.

The authors thank the participants of Economic Theory Workshop at Institute for Advanced Study (Princeton), GRIPS Workshop on Global Games (Tokyo), and Paris Workshop on Game Theory and Microeconomic Theory Seminar at Princeton University and at Rutgers University. Helpful conversations and comments from Satoru Takahashi, Stephen Morris, Richard P. McLean, Daisuke Oyama, Sylvain Chassang, and Marcin Peski are gratefully acknowledged. All remaining errors are, of course, solely by the authors. 


\section{Introduction}

In this paper we examine a dynamic strategy adjustment process in which players employ the log-linear stochastic choice rule. Players are assumed to be myopic and adjust their strategies at discrete times in response to a (distribution of) strategy profile prevailing in the previous period.

The log linear choice rule is so called because the log likelihood ratio of choosing one action over another, given other players' actions, is linearly proportional to the difference of payoffs they yield. The factor of proportionality is a crucial variable that parameterizes the process ranging from the uniform choice rule to a deterministic best-response rule. Thus the log-linear rule can be regarded as a perturbation of a best-response rule and it is indeed this view that we take here. Our main aim is to characterize the long-run behavior, stochastic stable states, of this process for a wider class of games than the existing literature when the process is close to the one under a best-response rule.

A more intuitive description of the log-linear process is that, the better an action (yielding a higher payoff), the exponentially more likely that it will be chosen. This is in a prominent contrast to other perturbed best-response processes (e.g., [18], [10]) ${ }^{1}$ in which all "mistakes" are equally likely. While the long-run behavior of the log-linear process and processes with uniformly likely mistakes agree in selecting the (weakly) risk-dominant equilibrium in two-player two action symmetric games with two strict equilibria in pure actions, their similarity ends there. ${ }^{2}$

One of the existing results that distinguishes the log-linear process from other perturbed best-response models is on the class of potential games $([12])$ due to $[2,3]$. If the game being played is a potential game, then the strategy profile(s) that maximizes its potential function will be observed almost always in the long run under any log-linear process that is sufficiently close to a best-response process. While useful in certain economic applications, the class of potential games is rather small. ${ }^{3}$ Our main result extends

\footnotetext{
${ }^{1}[10]$ studies perturbed Darwinian process. A Darwinian rule is any deterministic rule according to which a better action is better represented in the next generation.

${ }^{2}$ The weakly risk-dominant equilibrium is stochastically stable under the process of [18] for the same class $2 \times 2$ games but without requiring symmetry.

${ }^{3}$ Indeed, generically a game is not a potential game.
} 
the characterization of the long run behavior of the log linear-process beyond the class of potential games. ${ }^{4}$

The notion of potential game has been generalized in several directions. Weighted potential games are discussed in [12]. ${ }^{5}$ In a recent paper [1] provides an example of a weighted potential game for which the unique stochastically stable state under the loglinear dynamics differs from the action profile that maximizes the potential function. (See Example 5.) An important source of this phenomenon is that multiplying payoffs by a constant would also multiply the difference between payoffs (by the same constant) and hence the log-likelihood ratio of transition probabilities. Thus different weights (on a common potential function) for different players may result in a dynamic process for which maximizers of (unweighted) potential function are not stochastically stable. This example makes clear the strong cardinal nature of the log-linear choice rule and points us to a type of notion that generalizes the potential games and functions yet preserves the cardinality of payoff differences.

One possibility is to consider games in which payoff difference between two actions of any player is bounded by the corresponding difference in a common function. Concepts of this type were introduced and extensively discussed in connection to robustness of equilibria against slight incomplete information as an approach to equilibrium selection. (See, e.g., [8], [13], [6], [17] and [15].) Among them is a version of local potential function and local potential maximizer due to [13], later generalized in [15]) and this is the central concept employed in this paper.

A precise definition of local potential function and local potential maximizer will be given in Section 3. Here we will discuss a special case which illuminate the nature of local potential and its connection to log-linear process. A strategy profile, say $s^{*}=\left(s_{1}^{*}, \ldots, s_{I}^{*}\right)$, of a game in strategic form is said to be a local potential maximizer if one can find (a) a (total) order on each player's strategy set with $s_{i}^{*}$ being the unique maximum, and (b) a real valued function, say $v$, defined on the set of strategy profiles with two properties.

\footnotetext{
${ }^{4}$ Our characterization, however, should not be regarded as a strict generalization of the result on potential games. See Section 7.

${ }^{5}$ In fact, (exact) potential games are introduced as special cases of weighted potential games which in turn are special cases of ordinal potential game.
} 
First, $s^{*}$ is the unique maximizer of $v$. Second, if one action of a player is greater than another in the associated order, then the value of his payoff from the former action minus the payoff from the latter action, given any of others' strategies, is bounded from below by the corresponding difference in the values of $v$.

We already know the stochastically stable state of the log-linear process for a common interest game (potential game) where every player has an identical payoff function $v$. It is $s^{*}$. Now the second requirement above implies that whenever the log likelihood ratio of the log-linear choice probabilities between two actions in the common interest game is positive (resp. negative) so is the corresponding log likelihood ratio for the original game. Therefore, the two log-linear dynamics, one for $v$ and the other for the original payoff functions, moves (stochastically) in the same direction, i.e., towards $s^{*}$.

This simple observation suggests that the local potential maximizer, $s^{*}$, should also be stochastically stable under the original payoff functions. We will show that this is indeed the case but under an additional condition that either the original payoff functions or the function $v$ is supermodular. As the reader will see, supermodularity is needed to preserve the stochastic ordering of the two processes when one considers higher order transitions.

The above observation also suggests a line of the proof: compare (in a stochastic order) the two Markov processes. Indeed our proof uses only elementary tools of finite Markov chains. This is in a marked contrast to the methods employed in other works in the literature, i.e., (a finite version of) the stochastic potential/tree surgery technique of [7]. In a closely related work, [1] does employ this method. They provide an explicit formula for the stochastic potential for the log-linear process as well as associated radius-coradius results in the spirit of [5]. These results are applicable to any class of games and it is an interesting open question whether our results can be obtained with this method as easily. ${ }^{6}$ A potential advantage of our methods, though arguably not universally applicable, is that once stochastically stable states are known for one class of games, the results for another class of games may be obtained by comparing the associated Markov chains. In addition, finding a local potential maximizer is rather easy. Indeed, we provide a necessary and

\footnotetext{
${ }^{6}$ Especially when the population or a local interaction version of the game is considered in which the state space is rather large. Our method almost trivially carries over to such setting. See Section 6 . We have verified that the results on potential games can indeed be obtained by this method with relative ease.
} 
sufficient condition for a game to have a local potential maximizer. This condition refers only to the payoff functions of the game and computationally easy to verify. This is in stark contrast with the stochastic potential/tree surgery technique developed in [7] and applied to the log-linear dynamics by [1] which is known to be computationally difficult. ${ }^{7}$

As mentioned above, having an extension of potential maximizer that keeps track of payoff differences is crucial to obtain the results of our type for the log-linear processes. A more general, belief-based, notion of local potential maximizer has been introduced by [15]. A similar but distinct notion of monotone potential maximizer, based on properties of the best reply correspondence, is also given in the same work. ${ }^{8}$ These are in turn closely related to the concept of $\mathbf{p}$-dominance, a generalization of risk dominance ([4], [8]). Equilibrium selection results have been obtained for these concepts in various contexts, e.g., global games ([6]), robustness to incomplete information ([8], [15]), perfect foresight dynamics $([16]) .{ }^{9}$ We demonstrate by a series of examples that none of these weaker concepts guarantees stochastic stability under the log-linear dynamics. In particular, pdominance (hence monotone potential maximizer) is in general not sufficient for stochastic stability.

One reason for the lack of connection between these concepts and stochastic stability is that, as we alluded to above, the log-linear choice rule is sensitive to affine transformations of payoffs. Motivated by this observation, we modify the log-linear dynamics by incorporating a notion of relative, rather than absolute, payoff differences. More precisely, we define the relative log-linear choice rule under which the log likelihood ratio of choosing one action over another, given other players' actions, is linearly proportional to the relative difference of payoffs they yield. This choice rule is invariant with respect to affine transformations of payoffs. We show that in $2 \times 2$ games with two strict Nash equilibria, the weighted version of local potential maximizers is stochastically stable under this modified

\footnotetext{
${ }^{7}$ See [18] and references therein for some aspects of computational complexity of this method.

${ }^{8}$ Both local and monotone potential maximizers are - under the usual monotonicity conditions described in this paper - special cases of yet more general concept, generalized potential. See [15] for details.

${ }^{9} \mathrm{~A}$ comparison technique similar in spirit to ours has been employed in [16]. They show, e.g., that a (strict) monotone potential maximizer is globally accessible (and linearly absorbing) under an additional condition of supermodularity identical to ours (Theorem 2). We make further comments on this dynamics in Section 7.
} 
dynamics. This in particular implies that in this class of games, p-dominant equilibria as well as weighted potential maximizers are stochastically stable.

The rest of the paper is organized as follows. The basic setup is laid in Section 2. The central concept of local potential is defined in Section 3. There we also provide a necessary and sufficient condition for a subset of strategy profiles to be a local potential maximizer with constant weights. We present our main result (Theorem 2) in Section 4. In this section we will also present a series of examples illustrating the main result as well as examples demonstrating stochastic stability (or the lack thereof) of related concepts such as weighted potential maximizer and p-dominant equilibrium. The last part of this section is devoted to the study of relative log-linear dynamics for $2 \times 2$ games. The proof of the main result is presented in Section 5. In Section 6 we present a population (or random matching) as well as a local interaction version of the log-linear dynamics and the corresponding results. Section 7 concludes the paper.

\section{The Basic Model}

Consider an $I$-person finite game in strategic form. The set of actions available to player $i=1, \ldots, I$ is $S_{i}$ and his payoff function is $u_{i}: S \rightarrow \mathbb{R}$ where $S=S_{1} \times \cdots \times S_{I}$.

The dynamic process under consideration runs in discrete time $t=0,1,2, \ldots$ and its state space is $S$. At $t=0$ a strategy profile is selected according to an initial distribution. At each subsequent period a single player is selected and is given an opportunity to revise her strategy according to a stochastic choice rule. The probability that player $i$ is given this opportunity is denoted by $\rho_{i}$. We assume $\rho_{i}>0$ for all $i$. Thus state can change from $s$ to $s^{\prime}$ if, and only if, $s^{\prime}=\left(s_{i}^{\prime}, s_{-i}\right)$ for some $i$ and $s_{i}^{\prime} \in S_{i}$.

In this article we study the log-linear stochastic choice rule according to which the log likelihood ratio between two actions is proportional to the difference between the payoffs from these actions. The factor of proportionality is a nonnegative real number denoted by $\beta$. Let $p_{i}\left(s_{i} \mid s: u_{i}, \beta\right)$ be the probability that player $i$ chooses $s_{i} \in S_{i}$ given a state $s \in S$. The log-linear stochastic choice rule is characterized by

$$
\ln \frac{p_{i}\left(s_{i}^{\prime \prime} \mid s: u_{i}, \beta\right)}{p_{i}\left(s_{i}^{\prime} \mid s: u_{i}, \beta\right)}=\beta\left(u_{i}\left(s_{i}^{\prime \prime}, s_{-i}\right)-u_{i}\left(s_{i}^{\prime}, s_{-i}\right)\right)
$$


for all $s \in S$ and $s_{i}^{\prime}, s_{i}^{\prime \prime} \in S_{i}$. Thus given a revision opportunity and a current state $s$, player $i$ is exponentially more likely to select $s_{i}^{\prime \prime}$ than $s_{i}^{\prime}$ whenever $s_{i}^{\prime \prime}$ is a better reply to $s_{-i}$ than $s_{i}^{\prime}$ is. Equivalently,

$$
p_{i}\left(s_{i}^{\prime} \mid s: u_{i}, \beta\right)=\frac{e^{\beta u_{i}\left(s_{i}^{\prime}, s_{-i}\right)}}{\sum_{s_{i}^{\prime \prime} \in S_{i}} e^{\beta u_{i}\left(s_{i}^{\prime \prime}, s_{-i}\right)}} .
$$

It is clear from (2.1) and (2.2) that the log-linear rule $p_{i}\left(\cdot \mid s: u_{i}, \beta\right)$ is simply the uniform distribution on $S_{i}$ when $\beta=0$, and it converges as $\beta \rightarrow \infty$ to the uniform distribution over the best responses against $s_{-i}$.

Note that we have taken $\beta$ to be common to all players. In a later section we will discuss the processes with $\beta$ 's varying across player positions. We will also discuss later a random matching version of the process.

The log-linear choice rules generate a (time-homogeneous) Markov chain on the set of strategy profiles $S$ where the transition probability from $s$ to $s^{\prime}$ is given by

$$
q_{s s^{\prime}}(u, \beta)=\sum_{i=1}^{I} \mathbf{I}\left(s_{-i}^{\prime}=s_{-i}\right) \rho_{i} p_{i}\left(s_{i}^{\prime} \mid s: u_{i}, \beta\right)
$$

where $u=\left(u_{1}, \ldots, u_{I}\right)$, and $\mathbf{I}$ is the indicator function. Let $Q(u, \beta)=\left(q_{s s^{\prime}}(u, \beta)\right)_{s, s^{\prime} \in S}$ be the resulting transition matrix. Transition matrix when each player uses the best response rule is denoted by $Q^{*}(u)$. As we noted above $Q(u, \beta) \underset{\beta \rightarrow \infty}{\longrightarrow} Q^{*}(u)$.

It is straightforward to see that the Markov chain associated with $Q(u, \beta)$ is irreducible. Hence it possesses a unique invariant distribution $\mu(u, \beta)=\left(\mu_{s}(u, \beta)\right)_{s \in S}$, i.e., the unique solution to $\mu Q(u, \beta)=\mu$ and $\left(I+Q(u, \beta)+\cdots+Q(u, \beta)^{t}\right) /(t+1)$ converges as $t \rightarrow \infty$ to a matrix whose rows are identical to $\mu(u, \beta)$. So $\mu_{s}(u, \beta)$ is also the asymptotic average frequency with which state $s$ is visited. In addition, it is easy to verify that this chain is aperiodic and hence $Q(u, \beta)^{t}$ also converges to the matrix with rows equal to $\mu(u, \beta)$. In contrast the chain associated with $Q^{*}(f)$ typically has multiple recurrence classes and hence it possesses more than one invariant distribution. A well known result states that $\lim _{\beta \rightarrow \infty} \mu(u, \beta)$ exists and is an invariant distribution of the chain associated with $Q^{*}(f)$ (e.g., [18]). 
Definition 1 A state $s \in S$ is stochastically stable if $\lim _{\beta \rightarrow \infty} \mu_{s}(u, \beta)>0$.

Thus states that are not stochastically stable will be observed with a vanishing frequency in the long run under a log-linear process that is sufficiently close to the best response process, i.e., any log-linear process with large enough $\beta$.

\section{Local Potential}

In this section we discuss the main concept used in this paper: local potential (local function and local potential maximizer, to be more precise). It generalizes the concept of potential due to [12].

\subsection{Potential Games}

Recall that a game $\left(S_{i}, u_{i}\right)_{i=1, \ldots, I}$, as described above, is a potential game if there exists a function (potential function) $v: S \rightarrow \mathbb{R}$ with the property that

$$
u_{i}\left(s_{i}^{\prime}, s_{-i}\right)-u_{i}\left(s_{i}, s_{-i}\right)=v\left(s_{i}^{\prime}, s_{-i}\right)-v\left(s_{i}, s_{-i}\right)
$$

for all $i, s_{i}, s_{i}^{\prime}$ and $s_{-i}$.

For a potential game the log-linear process is a reversible Markov chain and its invariant distribution can be explicitly obtained by solving the detailed balance condition, $\mu_{s} q_{s s^{\prime}}(v, \beta)=\mu_{s^{\prime}} q_{s^{\prime} s}(v, \beta) .{ }^{10}$ Consequently, the set of stochastically stable states can be explicitly characterized.

Theorem $1([\mathbf{2}, \mathbf{3}])$ Suppose that $\left(S_{i}, u_{i}\right)_{i=1, \ldots, I}$ is a potential game with a potential function $v$. Then the invariant distribution of $Q(v, \beta)$ is

$$
\mu_{s}(v, \beta)=\frac{e^{\beta v(s)}}{\sum_{s^{\prime} \in S} e^{\beta v\left(s^{\prime}\right)}}
$$

and $s \in S$ is stochastically stable if, and only if, $s$ maximizes $v$.

\footnotetext{
${ }^{10}$ We write $q_{s s^{\prime}}(v, \beta), Q(v, \beta)$ etc. for the log-linear processes where $u_{i}=v$ for every $i=1, \ldots, I$.
} 


\subsection{Local Potential on an Ordered Domain}

The original definition of local potential appears in [13] where a local potential maximizer is defined as a single action profile that maximizes a local potential function. We employ a version of local potential due to [15] that directly generalizes that in [13]. In this version, a local potential function is defined to be a measurable function on the set of action profiles $S$ endowed with an algebra as explained below. A local potential maximizer is then defined to be the unique element of the algebra on which the local potential function attains the maximum. ${ }^{11}$ We will provide several examples of local potential maximizers in the next section.

An ordered domain on $S$ consists of, for each $i=1, \ldots, I$, a partition of $S_{i}$, denoted by $\left\{S_{i 1}, \ldots, S_{i K_{i}}\right\}$, and a partial order $\leq_{i}$ on $S_{i}$ where $s_{i} \leq_{i} s_{i}^{\prime}$ if $s_{i}=s_{i}^{\prime}$ or $s_{i} \in S_{i k}$ and $s_{i}^{\prime} \in S_{i k^{\prime}}$ with $k<k^{\prime}$. We write $s_{i}<_{i} s_{i}^{\prime}$ in the latter case. Let $\mathcal{S}_{i}$ be the algebra on $S_{i}$ generated by $\left\{S_{i 1}, \ldots, S_{i K_{i}}\right\}$. We define a partial order $\leq$ on $S$ by $s=\left(s_{1}, \ldots, s_{I}\right) \leq$ $s^{\prime}=\left(s_{1}^{\prime}, \cdots, s_{I}^{\prime}\right)$ if $s_{i} \leq_{i} s_{i}^{\prime}$ for all $i$. For each collection of $I$ integers $k_{1}, \ldots, k_{I}$ with $0 \leq k_{i} \leq K_{i}$, we let $S_{k_{1}, \ldots, k_{I}}=S_{1 k_{1}} \times \cdots \times S_{I k_{I}}$ and call a set of this form a measurable rectangle. Clearly, the family of measurable rectangles form a partition of $S$. Let $\mathcal{S}$ be the algebra on $S$ generated by this family. For each $i$ the partial order $\leq_{-i}$ and the algebra $\mathcal{S}_{-i}$ on $S_{-i}$ are similarly defined.

Definition 2 A set $S^{*} \subset S$ is a local potential maximizer (LP-max) with respect to the payoff functions $u=\left(u_{1}, \ldots, u_{I}\right)$ if there exist

(1) an ordered domain on $S:\left\{S_{i 1}, \ldots, S_{i K_{i}}\right\}, \leq_{i}, i=1, \ldots, I$

(2) a function $v: S \rightarrow \mathbb{R}$ (a local potential function), and

(3) a collection of nonnegative numbers $\left\{w_{i}\left(s_{i}, s_{i}^{\prime}\right) \mid s_{i} \leq_{i} s_{i}^{\prime}\right\}$ (weights) for each $i=1, \ldots, I$ such that

(a) $S^{*}$ is a measurable rectangle, $S^{*}=S_{k_{1}^{*}, \ldots, k_{I}^{*}}$,

(b) $v$ is $\mathcal{S}$-measurable, i.e., $v$ is constant on each measurable rectangle,

\footnotetext{
${ }^{11}$ In [15] a local potential on an ordered domain is defined as a special case of generalized potential. They discuss how a payoff-difference based definition of local potential employed by us in this paper implies their "belief based" definition.
} 
(c) $\operatorname{argmax} v=S^{*}$,

(d) for every $i$ and every $s_{-i} \in S_{-i}$,

(d-1) if $k<k_{i}^{*}, s_{i} \in S_{i k}$ and $s_{i}^{\prime} \in S_{i k+1}$, then

$$
w_{i}\left(s_{i}, s_{i}^{\prime}\right)\left(v\left(s_{i}^{\prime}, s_{-i}\right)-v\left(s_{i}, s_{-i}\right)\right) \leq u_{i}\left(s_{i}^{\prime}, s_{-i}\right)-u_{i}\left(s_{i}, s_{-i}\right),
$$

(d-2) if $k_{i}^{*}<k, s_{i} \in S_{i k-1}$ and $s_{i}^{\prime} \in S_{i k}$, then

$$
w_{i}\left(s_{i}, s_{i}^{\prime}\right)\left(v\left(s_{i}^{\prime}, s_{-i}\right)-v\left(s_{i}, s_{-i}\right)\right) \geq u_{i}\left(s_{i}^{\prime}, s_{-i}\right)-u_{i}\left(s_{i}, s_{-i}\right) .
$$

In order to show that a subset $S^{*} \subset S$ is a local potential maximizer, one must first identify an appropriate partition on each $S_{i}$ and the induced algebra on $S$ which includes $S^{*}$ as an element, then find a local potential function which is maximized on $S^{*}$.

It is easy to verify ([15], Lemma 8$)$ that if $S_{k_{1}^{*}, \ldots, k_{I}^{*}}$ is an LP-max with a local potential $v$, then for every player $i=1, \ldots, I$,

(i) for every $k<k_{i}^{*}$ and every $\sigma_{-i} \in \Delta\left(S_{-i}\right)$ such that $v\left(s_{i}, \sigma_{-i}\right) \leq v\left(s_{i}^{\prime}, \sigma_{-i}\right)$ for any $s_{i} \in S_{i k}$ and $s_{i}^{\prime} \in S_{i k+1}$, we have

$$
\max _{s_{i} \in S_{i k}} u_{i}\left(s_{i}, \sigma_{-i}\right) \leq \max _{s_{i}^{\prime} \in S_{i k+1}} u_{i}\left(s_{i}^{\prime}, \sigma_{-i}\right)
$$

(ii) for every $k_{i}^{*}<k$ and every $\sigma_{-i} \in \Delta\left(S_{-i}\right)$ such that $v\left(s_{i}^{\prime}, \sigma_{-i}\right) \geq v\left(s_{i} . \sigma_{-i}\right)$ for any $s_{i}^{\prime} \in S_{i k-1}$ and $s_{i} \in S_{i k}$, we have

$$
\max _{s_{i}^{\prime} \in S_{i k-1}} u_{i}\left(s_{i}^{\prime}, \sigma_{-i}\right) \geq \max _{s_{i} \in S_{i k}} u_{i}\left(s_{i}, \sigma_{-i}\right)
$$

In fact, in [15], an LP-max is defined to be the maximizer of a measurable function $v$ possessing the properties (i) and (ii). It can be shown ([15], Lemma 9) that if the partition on each $S_{i}$ is the finest, i.e. $\left\{\left\{s_{i}\right\} \mid s_{i} \in S_{i}\right\}$, then the two definitions are equivalent. ${ }^{12}$

For the main results of this paper, we are primarily interested in local potential maximizers with constant weights, in particular cases where weights $w_{i}\left(s_{i}, s_{i}^{\prime}\right)$ are independent of $\left(s_{i}, s_{i}^{\prime}\right)$ and of $i$, say $w_{i}(\cdot, \cdot) \equiv r$. Then, by renaming $r v$ as a new $v$, conditions (b) and (c) are satisfied, and condition (d) is equivalent to the following condition:

\footnotetext{
${ }^{12}[16]$ studies perfect foresight dynamics with a total order on each $S_{i}$, hence the finest partition on $S_{i}$.
} 
(e) For every $i$ and every $s_{-i} \in S_{-i}$,

(e-1) if $k<k^{\prime} \leq k_{i}^{*}$, then for any $s_{i} \in S_{i k}$ and $s_{i}^{\prime} \in S_{i k^{\prime}}$

$$
v\left(s_{i}^{\prime}, s_{-i}\right)-v\left(s_{i}, s_{-i}\right) \leq u_{i}\left(s_{i}^{\prime}, s_{-i}\right)-u_{i}\left(s_{i}, s_{-i}\right),
$$

(e-2) if $k_{i}^{*} \leq k<k^{\prime}$, then for any $s_{i} \in S_{i k}$ and $s_{i}^{\prime} \in S_{i k^{\prime}}$

$$
v\left(s_{i}^{\prime}, s_{-i}\right)-v\left(s_{i}, s_{-i}\right) \geq u_{i}\left(s_{i}^{\prime}, s_{-i}\right)-u_{i}\left(s_{i}, s_{-i}\right) .
$$

Thus we may as well take $w_{i}(\cdot, \cdot) \equiv 1$ in this case.

From Definition 2 it may seem difficult to see whether a given game admits an LP-max or how to find a local potential function. ${ }^{13}$ Below we provide a necessary and sufficient condition for a subset of strategy profiles to be an LP-max with constant weights (i.e. $w_{i}(\cdot, \cdot) \equiv 1$ for all $\left.i\right)$. The reader will see that the condition is easy to check and also yields the formula for a local potential function. ${ }^{14}$

To explain the condition in words first, let us consider for simplicity an ordered domain with the finest partition on each player's strategy set. In addition suppose that $s_{i}^{*}$ is the largest element in the given order on $S_{i}$. The necessary and sufficient condition for $s^{*}=\left(s_{1}^{*}, \ldots, s_{I}^{*}\right)$ to be an LP-max with constant weights is as follows. Take any other strategy profile $s$ and consider any sequence of strategy profiles starting at $s^{*}$, ending at $s$, and at each step one, and only one, player deviates to a strategy that is lower in the order than the previous one. If the sum of payoff differences for the deviating players along any such path is strictly positive, then, and only then, $s^{*}$ is an LP-max with constant weights. Below we generalize this condition to the set-valued LP-max which is not necessarily a product of the largest partition elements of each player's strategy sets.

Fix an ordered domain over $S,\left\{S_{i 1}, \ldots, S_{i K_{i}}\right\}, i=1, \ldots, I$. We will denote indices for measurable rectangles by bold letters, e.g., $\boldsymbol{k}=\left(k_{1}, \ldots, k_{I}\right)$ (of course, $\left.1 \leq k_{i} \leq K_{i}\right)$. We will also use notations such as $\left(k_{i}^{\prime}, \boldsymbol{k}_{-i}\right)$ in the usual manner.

\footnotetext{
${ }^{13}$ Though, given an ordered domain and a candidate for an LP-max, the problem is that of linear programming.

${ }^{14}$ The reader will also notice the similarity between our characterization of the LP-max and the conditions for a game to be a potential game provided by [12].
} 
For any pair of indices $\boldsymbol{k}=\left(k_{1}, \ldots, k_{I}\right)$ and $\boldsymbol{k}^{\prime}$ such that $\boldsymbol{k}^{\prime}=\left(k_{i}^{\prime}, \boldsymbol{k}_{-i}\right)$ for some $i$ and $k_{i}^{\prime} \neq k_{i}$, we define

$$
\Delta\left(\boldsymbol{k}, \boldsymbol{k}^{\prime}\right)=\min \left[u_{i}\left(s_{i}, s_{-i}\right)-u_{i}\left(s_{i}^{\prime}, s_{-i}\right)\right]
$$

where min is taken over all $s_{i} \in S_{i k_{i}}, s_{i}^{\prime} \in S_{i k_{i}^{\prime}}$ and $s_{-i} \in S_{\boldsymbol{k}_{-i}}$. Thus $\Delta\left(\boldsymbol{k}, \boldsymbol{k}^{\prime}\right)$ is the smallest gain (or the largest loss) to player $i$ when deviating unilaterally from some strategy profile in a rectangle $S_{\boldsymbol{k}}$ to another in $S_{\boldsymbol{k}^{\prime}}$. We say that a (finite) sequence of indices $\boldsymbol{\kappa}=\left(\boldsymbol{k}^{0}, \boldsymbol{k}^{1}, \ldots, \boldsymbol{k}^{L}\right)$ is a path of unilateral deviations if $\boldsymbol{k}^{\ell}=\left(k_{i}^{\ell}, \boldsymbol{k}_{-i}^{\ell-1}\right)$ for some $i$ and $k_{i}^{\ell} \neq k_{i}^{\ell-1}$ for each $\ell=1, \ldots, L$. For a path of unilateral deviations $\boldsymbol{\kappa}=\left(\boldsymbol{k}^{0}, \boldsymbol{k}^{1}, \ldots, \boldsymbol{k}^{L}\right)$ we set

$$
\Lambda(\boldsymbol{\kappa})=\sum_{\ell=1}^{L} \Delta\left(\boldsymbol{k}^{\ell-1}, \boldsymbol{k}^{\ell}\right)
$$

We say that a path of unilateral deviations $\boldsymbol{\kappa}=\left(\boldsymbol{k}^{0}, \boldsymbol{k}^{1}, \ldots, \boldsymbol{k}^{L}\right)$, where $\boldsymbol{k}^{\ell}=\left(k_{1}^{\ell}, \ldots, k_{I}^{\ell}\right)$, is individually monotonic if $k_{i}^{0} \geq k_{i}^{1} \geq \cdots \geq k_{i}^{L}$ or $k_{i}^{0} \leq k_{i}^{1} \leq \cdots \leq k_{i}^{L}$ for all $i$. The set of all individually monotonic paths of unilateral deviations starting at $\boldsymbol{k}\left(\boldsymbol{k}^{0}=\boldsymbol{k}\right)$ and ending at $\boldsymbol{k}^{\prime}\left(\boldsymbol{k}^{L}=\boldsymbol{k}^{\prime}\right)$ is denoted by $\Pi\left(\boldsymbol{k}, \boldsymbol{k}^{\prime}\right)$.

Proposition $1 S^{*} \subset S$ is a local potential maximizer with respect to $u=\left(u_{1}, \ldots, u_{n}\right)$ and with $w_{i}(\cdot, \cdot) \equiv 1$ for all $i=1, \ldots, n$ if, and only if,

(a) there exists an ordered domain $\left\{S_{i 1}, \ldots, S_{i K_{i}}\right\}, i=1, \ldots, I$, such that $S^{*}$ is a measurable rectangle: $S^{*}=S_{\boldsymbol{k}^{*}}$, and

(b) $\Lambda(\boldsymbol{\kappa})>0$ for every $\boldsymbol{\kappa} \in \Pi\left(\boldsymbol{k}^{*}, \boldsymbol{k}\right), \boldsymbol{k} \neq \boldsymbol{k}^{*}$.

In addition, under (a) and (b), the function $v: S \rightarrow \mathbb{R}$ defined by

$$
v(s)=\left\{\begin{array}{cl}
0 & \text { if } s \in S_{\boldsymbol{k}^{*}}, \\
-\min _{\boldsymbol{\kappa} \in \Pi\left(\boldsymbol{k}^{*}, \boldsymbol{k}\right)} \Lambda(\boldsymbol{\kappa}) & \text { if } s \in S_{\boldsymbol{k}}, \boldsymbol{k} \neq \boldsymbol{k}^{*}
\end{array}\right.
$$

provides an appropriate local potential function.

The proof of this proposition is given in Appendix A. We will utilize the proposition in the examples of the next section. 


\section{Stochastic Stability of Local Potential Maximizer}

We first state our main result.

Theorem 2 Suppose that $S_{k_{1}^{*}, \ldots, k_{n}^{*}}$ is a local potential maximizer with respect to $u=$ $\left(u_{1}, \ldots, u_{n}\right)$ with a local potential $v: S \rightarrow \mathbb{R}$ and $w_{i}(\cdot, \cdot) \equiv 1$ for all $i=1, \ldots, n$. If $u$ or $v$ is supermodular then $s \in S$ is stochastically stable with respect to the log-linear process only if $s \in S_{k_{1}^{*}, \ldots, k_{n}^{*}}$, i.e., the support of $\lim _{\beta \rightarrow \infty} \mu(u, \beta)$ is contained in $S_{k_{1}^{*}, \ldots, k_{n}^{*}}$.

To be clear, the supermodularity of $u_{i}$ or $v$ stated in the theorem is with respect to the partial orders on $S_{i}$ associated with an ordered domain which makes $S^{*}$ an LP-max. In particular, we say that $u_{i}$ is supermodular if

$$
u_{i}\left(s_{i}^{\prime}, s_{-i}\right)-u_{i}\left(s_{i}, s_{-i}\right) \leq u_{i}\left(s_{i}, s_{-i}^{\prime}\right)-u_{i}\left(s_{i}, s_{-i}^{\prime}\right)
$$

for all $s_{i}, s_{i}^{\prime} \in S_{i}$ with $s_{i}<s_{i}^{\prime}$ (i.e., $s_{i} \in S_{i k}, s_{i}^{\prime} \in S_{i k^{\prime}}$ with $k<k^{\prime}$ ) and all $s_{-i}, s_{-i}^{\prime} \in S_{-i}$ with $s_{-i}<s_{-i}^{\prime}$ (i.e., $s_{-i} \in S_{k_{-i}}, s_{i}^{\prime} \in S_{k_{-i}^{\prime}}$ with $k_{-i}<k_{-i}^{\prime}$ ).

We will present the proof of the theorem in the next section. The rest of this section is devoted to examples illustrating the theorem (Section (4.1)) and discussion of concepts related to local potential maximizer such as weighted potential ([12]) and p-dominance ([14]) (Section (4.2)). Motivated by examples in Section (4.2), we will consider in Section (4.3) the relative log-linear dynamics for $2 \times 2$ games and show that in contrast to the original log-linear dynamics, stochastically stable states are invariant to affine transformations of payoffs under this dynamics.

\subsection{Examples Illustrating the Main Theorem}

If the local potential maximizer is a singleton, then it is clearly the unique stochastically stable state provided, of course, that the conditions of the theorem are met.

Example 1 The $3 \times 3$ game on the left below appears in [18]. 


\begin{tabular}{c|c|c|c|}
\multicolumn{1}{c}{} & \multicolumn{1}{c}{0} & 1 & \multicolumn{1}{c}{2} \\
\cline { 2 - 4 } 0 & 6,6 & 0,5 & 0,0 \\
\cline { 2 - 4 } 1 & 5,0 & 7,7 & 5,5 \\
\cline { 2 - 4 } 2 & 0,0 & 5,5 & 8,8 \\
\hline
\end{tabular}

Game $u=\left(u_{1}, u_{2}\right)$

\begin{tabular}{c|c|c|c|}
\multicolumn{1}{c}{} & \multicolumn{1}{c}{0} & 1 & 2 \\
\cline { 2 - 4 } 0 & 6 & 5 & 0 \\
\cline { 2 - 4 } 1 & 5 & 7 & 5 \\
\cline { 2 - 4 } 2 & 0 & 5 & 8 \\
\cline { 2 - 4 } & &
\end{tabular}

Local potential $v$

It is easy to verify that the function $v: S \rightarrow \mathbb{R}$ exhibited on the right matrix is a local potential function for $u$ with weights $w_{i}(\cdot, \cdot) \equiv 1$ for $i=1,2$. The strategy pair $(2,2)$ is the unique local potential maximizer relative to the ordered domain with the finest partition $\left\{S_{i 1}, S_{i 2}, S_{i 3}\right\}=\{\{0\},\{1\},\{2\}\}, i=1,2$. In addition, $v$ is supermodular. Therefore, $(2,2)$ is the unique stochastically stable state under the log-linear process. In contrast, [18] has shown that $(1,1)$ is stochastically stable in his version of adaptive learning process.

In the next example, the set of stochastically stable states coincides with the local potential maximizer that is not a singleton.

Example 2 Consider a $3 \times 3$ game below. Note that this game is not a potential game in the sense of [12] as there is a best response cycle.

\begin{tabular}{c|c|c|c|}
\multicolumn{1}{c}{} & \multicolumn{1}{c}{0} & 1 & 2 \\
\cline { 2 - 4 } 0 & 1,1 & 0,0 & 0,0 \\
\cline { 2 - 4 } 1 & 0,0 & 3,2 & 2,3 \\
\cline { 2 - 4 } 2 & 0,0 & 2,3 & 3,2 \\
\cline { 2 - 4 } & & &
\end{tabular}

Game $u=\left(u_{1}, u_{2}\right)$

\begin{tabular}{l|l|l|l|}
\multicolumn{1}{c}{} & \multicolumn{1}{c}{0} & \multicolumn{1}{c}{1} & 2 \\
\cline { 2 - 4 } 0 & 1 & 0 & 0 \\
\cline { 2 - 4 } 1 & 0 & 2 & 2 \\
\cline { 2 - 4 } 2 & 0 & 2 & 2 \\
\cline { 2 - 4 } & & &
\end{tabular}

Local potential $v$

It is easy to check that $\{1,2\} \times\{1,2\}$ is an LP-max with constant weights, a local potential function given on the right matrix and an ordered domain $\left\{S_{i 1}, S_{i 2}\right\}=\{\{0\},\{1,2\}\}$, $i=1,2$. Note that with respect to this ordered domain, each $u_{1}$ and $u_{2}$ as well as the local potential function $v$ are supermodular. It is also easy to see that $\{1,2\} \times\{1,2\}$ is a recurrent class of the best response dynamics. Hence the set of stochastically stable states is precisely $\{1,2\} \times\{1,2\}$. 
The set of stochastically stable states may be a proper subset of the local potential maximizer. It is easy to see that the local potential maximizer is closed under unilateral best response deviations and hence it contains a recurrent class of the best response dynamics. But the local potential maximizer can contain a transient state for the best response dynamics, i.e., a strategy profile such that no path of unilateral best response deviation starting from it ever comes back to it. If the local potential maximizer contains a unique recurrent class, then stochastically stable states are precisely those belonging to that recurrent class.

Example 3 This is the cyclic matching pennies due to [8].

\begin{tabular}{c|c|c|c|}
\multicolumn{1}{c}{0} & \multicolumn{1}{c}{1} & 2 \\
\cline { 2 - 4 } 0 & $1,1,1$ & $0,0,0$ & $0,0,0$ \\
\cline { 2 - 4 } 1 & $0,0,0$ & $0,0,0$ & $0,0,0$ \\
\cline { 2 - 4 } 2 & $0,0,0$ & $0,0,0$ & $0,0,0$ \\
\cline { 2 - 4 } & &
\end{tabular}

0

\begin{tabular}{c|c|c|c|}
\multicolumn{1}{c}{0} & \multicolumn{1}{c}{1} & 2 \\
\cline { 2 - 4 } 0 & $0,0,0$ & $0,0,0$ & $0,0,0$ \\
\cline { 2 - 4 } 1 & $0,0,0$ & $2,2,2$ & $2,3,3$ \\
\cline { 2 - 4 } 2 & $0,0,0$ & $3,3,2$ & $3,2,3$ \\
\cline { 2 - 4 }
\end{tabular}

1

\begin{tabular}{c|c|c|c|}
\multicolumn{1}{c}{0} & \multicolumn{1}{c}{1} & \multicolumn{1}{c}{2} \\
\cline { 2 - 4 } 0 & $0,0,0$ & $0,0,0$ & $0,0,0$ \\
\cline { 2 - 4 } 1 & $0,0,0$ & $3,2,3$ & $3,3,2$ \\
\cline { 2 - 4 } 2 & $0,0,0$ & $2,3,3$ & $2,2,2$ \\
\cline { 2 - 4 } & &
\end{tabular}

2

$$
\text { Game } u=\left(u_{1}, u_{2}, u_{3}\right)
$$

\begin{tabular}{l|l|l|l|}
\multicolumn{1}{c}{} & \multicolumn{1}{c}{0} & 1 & 2 \\
\cline { 2 - 4 } 0 & 1 & 0 & 0 \\
\cline { 2 - 4 } 1 & 0 & 0 & 0 \\
\cline { 2 - 4 } 2 & 0 & 0 & 0 \\
\cline { 2 - 4 } & 0 & \multicolumn{3}{c}{0}
\end{tabular}

\begin{tabular}{l|l|l|l|}
\multicolumn{1}{c}{} & \multicolumn{1}{c}{0} & \multicolumn{1}{c}{1} & 2 \\
\cline { 2 - 4 } 0 & 0 & 0 & 0 \\
\cline { 2 - 4 } 1 & 0 & 2 & 2 \\
\cline { 2 - 4 } 2 & 0 & 2 & 2 \\
\cline { 2 - 4 } & &
\end{tabular}

1

\begin{tabular}{c|l|l|l|}
\multicolumn{1}{c}{} & \multicolumn{1}{c}{0} & \multicolumn{1}{c}{1} & 2 \\
\cline { 2 - 4 } 0 & 0 & 0 & 0 \\
\cline { 2 - 4 } 1 & 0 & 2 & 2 \\
\cline { 2 - 4 } 2 & 0 & 2 & 2 \\
\cline { 2 - 4 } & 0 & \multicolumn{3}{|c}{2}
\end{tabular}

Local potential $v$

The payoff functions $u_{i}, i=1,2,3$, are supermodular. With respect to an ordered domain $\left\{S_{i 1}, S_{i 2}, S_{i 3}\right\}=\{\{0\},\{1,2\}\}, i=1,2,3$, it is easy to verify that the product set $\{1,2\} \times\{1,2\} \times\{1,2\}$ is a local potential maximizer with constant weights and a local potential function $v$ as above. ${ }^{15}$ However, within this set $(1,1,1)$ and $(2,2,2)$ are clearly

\footnotetext{
${ }^{15}$ It can be shown that there is no singleton action profile that is an LP-max even with non constant weights. See [8].
} 
transient states for the best response process. Hence the set of stochastically stable states is $(\{1,2\} \times\{1,2\} \times\{1,2\}) \backslash\{(1,1,1),(2,2,2)\}$.

As an application of the main theorem to a class of games, we next consider a version of unanimity games. ${ }^{16}$ The payoff functions of a unanimity game are supermodular. Using Proposition 1, we will provide a necessary and sufficient condition for a unanimous agreement to be a singleton LP-max with constant weights and hence stochastically stable.

Example 4 Each player has two actions, $S_{i}=\{0,1\}$. Let $\mathbf{0}=(0, \ldots, 0)$ and $\mathbf{1}=$ $(1, \ldots, 1)$. The payoffs are such that $u_{i}(\mathbf{0})>0, u_{i}(\mathbf{1})>0$ and $u_{i}(s)=0$ for all $s \neq \mathbf{0}, \mathbf{1}$. Thus there are two strict equilibria, $\mathbf{0}$ and $\mathbf{1}$. Note that each $u_{i}$ is supermodular.

Claim 1 The singleton set $\{\mathbf{1}\}$ (resp. $\{\mathbf{0}\})$ is an LP-max with constant weights if, and only if $u_{i}(\mathbf{1})>u_{j}(\mathbf{0})\left(\right.$ resp. $\left.u_{i}(\mathbf{0})>u_{j}(\mathbf{1})\right)$ for all $i$ and $j \neq i$. Consequently, if $u_{i}(\mathbf{1})>$ $u_{j}(\mathbf{0})$ (resp. $\left.u_{i}(\mathbf{0})>u_{j}(\mathbf{1})\right)$ for all $i$ and $j \neq i$, then $s=\mathbf{1}$ (resp. $\left.s=\mathbf{0}\right)$ is stochastically stable.

Proof. First note that since $\{\mathbf{1}\}$ must be a measurable rectangle, the only candidate for the ordered domain over $S$ is the finest partition. Without loss of generality let $\left\{S_{i 1}, S_{i 2}\right\}=\{\{0\},\{1\}\}, i=1, \ldots, I$. Set $\boldsymbol{k}^{*}=(2,2, \ldots, 2)$ so that $S_{\boldsymbol{k}^{*}}=\{\mathbf{1}\}$. Pick any individually monotonic path of unilateral deviations $\boldsymbol{\kappa}=\left(\boldsymbol{k}^{0}, \boldsymbol{k}^{1}, \ldots, \boldsymbol{k}^{L}\right)$ with $\boldsymbol{k}^{0}=\boldsymbol{k}^{*}$ and $\boldsymbol{k}^{L} \neq \boldsymbol{k}^{*}$. Let $\boldsymbol{k}^{1}=\left(k_{i}^{1}, \boldsymbol{k}_{-i}^{*}\right)\left(=\left(1, \boldsymbol{k}_{-i}^{*}\right)\right)$ and $\boldsymbol{k}^{L}=\left(k_{j}^{L}, \boldsymbol{k}_{-j}^{L-1}\right)$. Note that $\Delta\left(\boldsymbol{k}^{0}, \boldsymbol{k}^{1}\right)=u_{i}(\mathbf{1})$ and $\Delta\left(\boldsymbol{k}^{L-1}, \boldsymbol{k}^{L}\right)=-u_{j}(\mathbf{0})$ or 0 depending on $\boldsymbol{k}^{L}=(1,1, \ldots, 1)$ or not. Also, $\Delta\left(\boldsymbol{k}^{\ell-1}, \boldsymbol{k}^{\ell}\right)=0$ for $1<\ell<L$. Hence,

$$
\Lambda(\boldsymbol{\kappa})=\sum_{\ell=1}^{L} \Delta\left(\boldsymbol{k}^{\ell-1}, \boldsymbol{k}^{\ell}\right)= \begin{cases}u_{i}(\mathbf{1})-u_{j}(\mathbf{0}) & \text { if } \boldsymbol{k}^{L}=(1,1, \ldots, 1) \\ u_{i}(\mathbf{1}) & \text { otherwise }\end{cases}
$$

Note that if $\boldsymbol{k}^{L}=(1,1, \ldots, 1)$, then it must be that $j \neq i$. Since choices of $i$ and $j$ are arbitrary the conclusion follows from Proposition 1.

Q.E.D.

It follows from this claim that if one of the strict equilibria is an LP-max with constant weights, then it is strictly preferred to the other strict equilibrium by all but, possibly, one

\footnotetext{
${ }^{16}$ This class of games is also studied in [15] and [16].
} 
player. It is now straightforward to check that $\mathbf{1}=(1,1,1)$ is the LP-max with constant weights in the three-player unanimity game below.

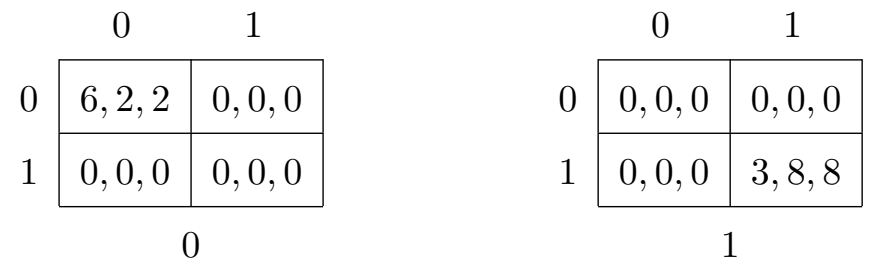

\subsection{Non-constant Weights, p-dominance}

One may wish to extend our main theorem to games with a local potential maximizer allowing for non-constant weights and, perhaps, with additional conditions such as $u$ exhibiting diminishing marginal returns. The following example, due to [1], shows that this is not possible.

Example 5 The 2-by-2 game on the left matrix is a weighted potential game where the potential function is given by $v$ on the right matrix with weights $w_{1}=1$ for player 1 and $w_{2}=\frac{1}{4}$ for player 2 .

\begin{tabular}{c|c|c|} 
& \multicolumn{1}{c}{0} & 1 \\
\cline { 2 - 3 } 0 & 2,2 & 0,0 \\
\cline { 2 - 3 } 1 & 0,0 & 10,1 \\
\hline \multicolumn{3}{l}{$u=\left(u_{1}, u_{2}\right)$}
\end{tabular}

\begin{tabular}{c|c|c|}
\multicolumn{1}{c}{0} & \multicolumn{1}{c}{0} & 1 \\
\cline { 2 - 3 } 0 & 2 & -6 \\
\cline { 2 - 3 } 1 & 0 & 4 \\
\cline { 2 - 2 } & $v$
\end{tabular}

The strategy pair $(1,1)$ uniquely maximizes $v$. With respect to the ordered domain $\left\{S_{i 1}, S_{i 2}\right\}=\{\{0\},\{1\}\}, i=1,2$, the singleton set $\{(1,1)\}$ is indeed a local potential maximizer where the local potential function is $v$ and the weights are $w_{1}(\cdot, \cdot) \equiv 1$ and $w_{2}(\cdot, \cdot) \equiv \frac{1}{4} \cdot{ }^{17}$ Using the tree surgery argument $([7] ;[18],[10])$, it has been shown in [1]

\footnotetext{
${ }^{17}$ It is easy to verify that there is no LP-max with constant weights for this game. For example, take any ordered domain with $\{(1,1)\}$ as a measurable rectangle, say, $\left\{S_{i 1}, S_{i 2}\right\}=\{\{0\},\{1\}\}, i=1,2$, so $S_{2,2}=$ $\{(1,1)\}$. For a path $\boldsymbol{\kappa}=((2,2),(2,1),(1,1)) \in \Pi((2,2),(1,1))$, we have $\Lambda(\kappa)=(1-0)+(0-2)=-1<0$.
} 
that the strategy pair $(0,0)$ uniquely minimizes the stochastic potential (see Appendix B) and hence it is the unique stochastically stable state.

From the proof of our main theorem in the next section, it can be easily verified that if we allow the parameter $\beta$ to vary across players, $\beta_{1}=\beta$ and $\beta_{2}=4 \beta$ for this example, then the potential maximizer becomes stochastically stable, i.e., $\lim _{\beta \rightarrow \infty} \mu_{(1,1)}(u,(\beta, 4 \beta))=1$. More generally, let $\mathbf{b}=\left(\beta_{1}, \ldots, \beta_{n}\right)$ where $\beta_{i} \geq 0$ and let $Q(u, \mathbf{b})$ be the transition matrix for the $\log$-linear process where the parameter $\beta$ for player $i$ 's stochastic choice rule $(2.1)$ (resp. (2.2)) is replaced by $\beta_{i}$. Again $Q(u, \mathbf{b})$ is irreducible and aperiodic, and its unique invariant distribution is denoted by $\mu(u, \mathbf{b})$.

Proposition 2 Suppose that $S^{*}$ is an LP-max with respect to $u=\left(u_{1}, \ldots, u_{I}\right)$ with a local potential $v: S \rightarrow \mathbb{R}$ and $w_{i}(\cdot, \cdot) \equiv w_{i}, i=1, \ldots, I$. If $u$ or $v$ is supermodular then $s$ is stochastically stable with respect to the log-linear process with $\mathbf{b}=\left(\frac{\beta}{w_{1}}, \ldots, \frac{\beta}{w_{I}}\right)$ only if $s \in S^{*}$, i.e., the support of $\lim _{\beta \rightarrow \infty} \mu(u, \mathbf{b})$ is contained in $S^{*}$.

The concept of $\mathbf{p}$-dominance ([8]) is related to (though distinct from) that of LP-max. Let $\mathbf{p}=\left(p_{1}, \ldots, p_{I}\right)$ with $0 \leq p_{i} \leq 1, i=1, \ldots, I$. A strategy profile $s^{*} \in S$ is a $\mathbf{p}$-dominant (resp. strict $\mathbf{p}$-dominant) equilibrium if, for every player $i, s_{i}^{*}$ is a (resp. the unique) best response to any mixture over $S_{-i}$ that puts probability at least (resp. strictly greater than) $p_{i}$ on $s_{-i}^{*}$. If $s^{*}$ is a $\mathbf{p}$-dominant equilibrium with $p_{1}+\cdots+p_{I}<1$, then the singleton set $\left\{s^{*}\right\}$ is an LP-max with respect to the ordered domain $\left\{S_{i 1}, S_{i 2}\right\}=\left\{S_{i} \backslash\left\{s_{i}^{*}\right\},\left\{s_{i}^{*}\right\}\right\}$ for each $i=1, \ldots, I$ and a local potential function

$$
v(s)= \begin{cases}1-\sum_{i=1}^{I} p_{i} & \text { if } s=s^{*} \\ -\sum_{i: s_{i}=s_{i}^{*}} p_{i} & \text { otherwise. }\end{cases}
$$

Hence, by Proposition 1, $S_{2,2}=\{(1,1)\}$ cannot be an LP-max with constant weights. Argument for other ordered domains is similar. One can verify by a similar argument that $\{(0,0)\}$ is not an LP-max with constant weights and hence its stochastic stability does not follow from our result. 
(Morris and Ui, 2005, Lemma 7) possibly with nonconstant weights. For the game of Example $5,(1,1)$ is a $\left(\frac{1}{6}, \frac{2}{3}\right)$-dominant equilibrium and hence $\{(1,1)\}$ is an LP-max with a local potential function given in the matrix below.

\begin{tabular}{c|r|r|}
\multicolumn{1}{c}{} & \multicolumn{1}{c}{0} & 1 \\
\cline { 2 - 3 } 0 & 0 & $-\frac{2}{3}$ \\
\cline { 2 - 3 } 1 & $-\frac{1}{6}$ & $\frac{1}{6}$ \\
\cline { 2 - 3 } & &
\end{tabular}

$v$

One can easily verify that the weights are $w_{1}(\cdot, \cdot) \equiv 12$ and $w_{2}(\cdot, \cdot) \equiv 3 .^{18}$

As we saw above, the unique stochastically stable state is $(0,0)$. Thus a strategy profile being $\mathbf{p}$-dominant with $p_{1}+\cdots+p_{I}<1$ is no guarantee for stochastic stability. ${ }^{19}$ For a $2 \times 2$ games, however, it can be shown, using the tree surgery argument, that $\left(p_{1}, p_{2}\right)$ dominance with $\max \left\{p_{1}, p_{2}\right\}<\frac{1}{2}$ is sufficient for stochastic stability. The proof of this fact is presented in Appendix B. The proof also shows that that if $s^{*}$ is a strict $\left(p_{1}, p_{2}\right)$ dominant equilibrium with $\max \left\{p_{1}, p_{2}\right\}<\frac{1}{2}$, then it is the unique stochastically stable state.

It should be remarked that the condition $\max \left\{p_{1}, p_{2}\right\}<\frac{1}{2}$ does not imply that a $\left(p_{1}, p_{2}\right)$-dominant equilibrium is an LP-max with constant weights as the next example demonstrates. $^{20}$

Example 6 Consider a $2 \times 2$ game given by

\footnotetext{
${ }^{18}$ This local potential function is an affine transformation of the one in Example 5: multiply by 12 and add 2 . The weights have been multiplied by 12 accordingly.

${ }^{19}$ In fact, $(1,1)$ is a strict $\left(\frac{1}{6}, \frac{2}{3}\right)$-dominant equilibrium. A strategy profile $s^{*}$ is a strict p-dominant equilibrium if, for each player $i, s_{i}^{*}$ is the unique best response to any mixed actions of others that put a probability strictly greater than $p_{i}$. So strict $\mathbf{p}$-dominance with $p_{1}+\cdots+p_{n}<1$ does not ensure stochastic stability, either.

${ }^{20}$ Again, adding strict p-dominance is of no help, either, as the equilibrium considered in the example is indeed strict $\left(p_{1}, p_{2}\right)$-dominant with $p_{1}, p_{2}<\frac{1}{2}$.
} 


\begin{tabular}{c|c|c|}
\multicolumn{1}{c}{0} & 1 \\
\cline { 2 - 3 } 0 & 2,2 & $0,-2$ \\
\cline { 2 - 3 } 1 & $0,-2$ & 3,3 \\
\cline { 2 - 2 } & \multicolumn{3}{c}{$u=\left(u_{1}, u_{2}\right)$}
\end{tabular}

Strategy profile $(1,1)$ is a strict $\left(\frac{2}{5}, \frac{4}{9}\right)$-dominant equilibrium and hence it is the unique stochastically stable state. However, $\{(1,1)\}$ cannot be an LP-max with constant weights. Indeed, if $\{(1,1)\}$ is an LP-max, the ordered domain must be the one with the fines partitions, say, $\left\{S_{i 1}, S_{i 2}\right\}=\{\{0\},\{1\}\}, i=1,2$. For the path $\boldsymbol{\kappa}=((2,2),(1,2),(1,1)) \in$ $\Pi((2,2),(1,1))$ we have $\Lambda(\boldsymbol{\kappa})=(3-0)+(-2-2)=-1<0$ and so by Proposition 1 , $\{(1,1)\}$ cannot be an LP-max with constant weights.

For $2 \times 2$ symmetric games, it can be shown that if $s^{*} \in S$ is a strict $\left(p_{1}, p_{2}\right)$-dominant equilibrium with $p_{1}+p_{2}<1$, then $\left\{s^{*}\right\}$ is a local potential maximizer with constant weights and a supermodular local potential function, and hence stochastically stable by Theorem 2. A risk-dominant equilibrium is a special case.

The next example shows that neither of the results for $2 \times 2$ games mentioned above can be extended to a larger class of games. Specifically, it shows (a) p-dominance with $p_{i}<\frac{1}{2}$ (or even $p_{i}<\frac{1}{\left|S_{-i}\right|}$ ) for all $i$ is not sufficient for stochastic stability, and (b) even for symmetric games, the same condition does not guarantee that the equilibrium is an LP-max with constant weights or stochastically stable.

Example 7 Consider a $3 \times 3$ symmetric game given by

\begin{tabular}{c|c|r|c|}
\multicolumn{1}{c}{} & \multicolumn{1}{c}{0} & \multicolumn{1}{c}{1} & \multicolumn{1}{c}{2} \\
\cline { 2 - 4 } 0 & $-1,-1$ & $-1,4$ & 3,2 \\
\cline { 2 - 4 } 1 & $4,-1$ & 2,2 & 0,0 \\
\cline { 2 - 4 } 2 & 2,3 & 0,0 & $x, x$ \\
\cline { 2 - 4 } & & &
\end{tabular}

where $4<x<5$. It is easily verified that $(2,2)$ is a $\left(\frac{2}{x+2}, \frac{2}{x+2}\right)$-dominant equilibrium. Note that $\frac{2}{x+2}<\frac{1}{3}$ as $4<x$. However, it is neither an LP-max (with constant weights) 
nor stochastically stable. The latter claim, as well as stochastic stability of $(1,1)$, can be demonstrated by the tree surgery argument as in [1]. (See also Appendix B).

To see that $\{(2,2)\}$ is not an LP-max with constant weights, take any ordered domain with $\{(2,2)\}$ is a measurable rectangle, say, $\left\{S_{i 1}, S_{i 2}, S_{i 3}\right\}=\{\{0\},\{1\},\{2\}\}, i=1,2$, so $S_{3,3}=\{(2,2)\}$. For a path $\boldsymbol{\kappa}=((3,3),(1,3),(1,2)) \in \Pi((3,3),(1,2))$, we have $\Lambda(\boldsymbol{\kappa})=$ $(x-3)+(2-4)=x-5<0$. Hence, by Proposition $1, S_{3,3}=\{(2,2)\}$ cannot be an LP-max with constant weights. Argument for other ordered domains is similar. One can verify by a similar argument that $\{(1,1)\}$ is not an LP-max with constant weights and hence its stochastic stability does not follow from out result.

This example also demonstrates that the set of stochastically stable states for the log-linear process is sensitive to the addition of strictly dominated actions. Indeed if we eliminate the strictly dominated action 0 for each player, then the resulting $2 \times 2$ game is a potential game and the potential maximizer $(2,2)$ is stochastically stable. As mentioned in the example, addition of a strictly dominated action 0 changes the unique stochastically stable state to $(1,1)$.

\subsection{The Relative Log-linear Dynamics}

Examination of examples in the last section reveals that the log-linear process introduced by $[2,3]$ is sensitive to affine transformations of payoffs i.e. such a transformation may affect the set of stochastically stable states. This lack of invariance is a significant reason why local potential maximizers with non-constant weights are generally not stochastically stable under this process. To illustrate this point formally we modify the log-linear stochastic choice rule (recall $(2.1),(2.2)$ ) by incorporating a notion of relative losses. Under the modified rule, called relative log-linear choice rule, the log likelihood ratio of choosing one action over another, given other players' actions, is linearly proportional to the relative difference of payoffs they yield. While studying this dynamics in full generality is beyond the scope of this paper, we study in this section its properties and relation to local potential maximizer in a class of 2-by-2 games: strategy sets are $S_{1}=S_{2}=\{0,1\}$ and payoff functions $\left(u_{1}, u_{2}\right)$ are such that $(0,0)$ and $(1,1)$ are both strict equilibria. 
The relative log-linear stochastic choice rule for players 1 is characterized by

$$
\ln \frac{p_{1}^{r}\left(1 \mid s: u_{1}, \beta\right)}{p_{1}^{r}\left(0 \mid s: u_{1}, \beta\right)}=\beta\left(\frac{u_{1}\left(1, s_{2}\right)-u_{1}\left(0, s_{2}\right)}{D_{1}}\right)
$$

where $D_{1}=\left[u_{1}(1,1)-u_{1}(0,1)\right]+\left[u_{1}(0,0)-u_{1}(1,0)\right]$ is the sum of the payoff losses when deviating from each equilibrium. Note that $D_{1}>0$ as $(0,0)$ and $(1,1)$ are assumed to be strict equilibria. Similarly, for player 2,

$$
\ln \frac{p_{2}^{r}\left(1 \mid s: u_{2}, \beta\right)}{p_{2}^{r}\left(0 \mid s: u_{2}, \beta\right)}=\beta\left(\frac{u_{2}\left(s_{1}, 1\right)-u_{2}\left(s_{1}, 0\right)}{D_{2}}\right)
$$

where $D_{2}=\left[u_{2}(1,1)-u_{2}(1,0)\right]+\left[u_{2}(0,0)-u_{2}(0,1)\right]>0$. We let $Q^{r}(u, \beta)$ be the resulting transition matrix and $\mu^{r}(u, \beta)$ its unique invariant distribution.

Note that, unlike the original log-linear choice rule (2.1), the log likelihood ratios (4.1) and (4.2) under the relative log-linear rule remain unchanged if we apply an affine transformations to the corresponding player's payoffs.

Given a payoff function $u_{i}, i=1,2$, define the relative payoff function of player $i$ by

$$
u_{i}^{r}(s)=\frac{u_{i}(s)}{D_{i}} .
$$

Note that

$$
\ln \frac{p_{i}^{r}\left(1 \mid s: u_{i}, \beta\right)}{p_{i}^{r}\left(0 \mid s: u_{i}, \beta\right)}=\ln \frac{p_{i}\left(1 \mid s: u_{i}^{r}, \beta\right)}{p_{i}\left(0 \mid s: u_{i}^{r}, \beta\right)}
$$

so the relative log-linear dynamics for the game $u=\left(u_{1}, u_{2}\right)$ is identical to the original log-linear dynamics for the "relative game" $u^{r}=\left(u_{1}^{r}, u_{2}^{r}\right)$. In particular we have

$$
\mu^{r}(u, \beta)=\mu\left(u^{r}, \beta\right) \quad \text { for every } \beta \geq 0 \text {. }
$$

For instance, it is straightforward to see that if $u=\left(u_{1}, u_{2}\right)$ is a weighted potential game, then $u^{r}=\left(u_{1}^{r}, u_{2}^{r}\right)$ is a (exact) potential game. Hence, given Theorem 1 , we can easily show that the maximizer of a potential function for $u$ is stochastically stable under the relative log-linear dynamics. Recall from Example 5 that under the original log-linear dynamics, a potential maximizer for a weighted potential game is not necessarily stochastically stable. More generally, we show below that if $u$ admits a local potential function with strictly positive weights, then $u^{r}$ admits a local potential function with constant weights with the same maximizer, which, by Theorem 2 , is stochastically stable. 
Proposition 3 If $\left\{s^{*}\right\}\left(s^{*} \in\{0,1\} \times\{0,1\}\right)$ is a local potential maximizer with respect to $u=\left(u_{1}, u_{2}\right)$ with strictly positive weights, then $s^{*}$ is the unique stochastically stable state under the relative log-linear process, i.e. $\operatorname{Support}\left(\lim _{\beta \rightarrow \infty} \mu^{r}(u, \beta)\right)=\left\{s^{*}\right\}$.

Proof. If the singleton set $\left\{s^{*}\right\}$ is a local potential maximizer, then it must be a strict equilibrium and so $s^{*}$ is either $(0,0)$ or $(1,1)$ by assumption. Let $s^{*}=(1,1)$. (The proof for the case $s^{*}=(0,0)$ is identical.) In this case, the ordered domain must be the one with the finest partitions. Without loss of generality consider $\left\{S_{i 1}, S_{i 2}\right\}=\{\{0\},\{1\}\}, i=1,2$. Let $v: S \rightarrow \mathbb{R}$ be the local potential function for $u=\left(u_{1}, u_{2}\right)$ with $(1,1)$ being the unique maximizer together with strictly positive weights ${ }^{21} w_{i}=w_{i}(0,1)>0, i=1,2$. We then have

$$
\begin{array}{ll}
w_{1}\left(v\left(1, s_{2}\right)-v\left(0, s_{2}\right)\right) \leq u_{1}\left(1, s_{2}\right)-u_{1}\left(0, s_{2}\right) & \text { for all } s_{2}=0,1 \\
w_{2}\left(v\left(s_{1}, 1\right)-v\left(s_{1}, 0\right)\right) \leq u_{2}\left(s_{1}, 1\right)-u_{2}\left(s_{1}, 0\right) & \text { for all } s_{1}=0,1
\end{array}
$$

Define $v^{r}: S \rightarrow \mathbb{R}$ by

$$
v^{r}(s)=\frac{v(s)}{[v(1,1)+v(0,0)]-[v(0,1)+v(1,0)]} .
$$

Note that because $\{(1,1)\}$ is the LP-max, we have $v(1,1)>v(0,1)$ and, since $(0,0)$ is a strict equilibrium by assumption,

$$
w_{1}(v(1,0)-v(0,0)) \leq u_{1}(1,0)-u_{1}(0,0)<0 .
$$

So $v(0,0)-v(1,0)>0$. Thus the denominator in the above expression for $v^{r}$ is strictly positive. Hence $\{(1,1)\}$ is also the unique maximizer of $v^{r}$.

We now show that in the game with relative payoffs $u^{r}=\left(u_{1}^{r}, u_{2}^{r}\right)$ as defined in (4.3), $\{(1,1)\}$ is an LP-max with constant weights and a local potential function $v^{r}$ as defined in (4.7). Since $u_{i}, i=1,2$ is supermodular ${ }^{22}$ with respect to the ordering $0<_{i} 1$, so is $u_{1}^{r}, i=1,2$. The desired conclusion then follows from Theorem 2 that implies

\footnotetext{
${ }^{21}$ Recall from Definition 2 that weights $w_{i}\left(s_{i}, s_{i}^{\prime}\right)$ are defined only for pairs of strategies with $s_{i} \leq_{i} s_{i}^{\prime}$.

${ }^{22}$ Since $(0,0)$ and $(1,1)$ are strict equilibria, we have $u_{1}(1,0)-u_{1}(0,0)<0<u_{1}(1,1)-u_{1}(0,1)$ and a similar inequality holds for $u_{2}$.
} 
$\operatorname{Support}\left(\lim _{\beta \rightarrow \infty} \mu\left(u^{r}, \beta\right)\right)=\{(1,1)\}$, and hence $\operatorname{Support}\left(\lim _{\beta \rightarrow \infty} \mu^{r}(u, \beta)\right)=\{(1,1)\}$ by (4.4).

We verify the necessary inequalities, Definition $2(3)-(d)$, for player 1 . The argument for player 2 is identical. Rewrite (4.5) explicitly:

$$
\begin{aligned}
& 0<w_{1}[v(1,1)-v(0,1)] \leq u_{1}(1,1)-u_{1}(0,1), \\
& 0<u_{1}(0,0)-u_{1}(1,0) \leq w_{1}[v(0,0)-v(1,0)] .
\end{aligned}
$$

We thus have

$$
\begin{aligned}
v^{r}(1,1)-v^{r}(0,1) & =\frac{w_{1}[v(1,1)-v(0,1)]}{w_{1}[v(1,1)-v(0,1)]+w_{1}[v(0,0)-v(1,0)]} & & \text { by }(4.7) \\
& \leq \frac{u_{1}(1,1)-u_{1}(0,1)}{\left[u_{1}(1,1)-u_{1}(0,1)\right]+\left[u_{1}(0,0)-u_{1}(1,0)\right]} & & \text { by }(4.8), \\
& =u_{1}^{r}(1,1)-u_{1}^{r}(0,1) & & \text { by }(4.3),
\end{aligned}
$$

This completes the proof.

Q.E.D.

Strictly positive weights in the statement of Proposition 3 can actually be dispensed with for the class of games under consideration. This is due to the fact that we assumed that $(1,1)$ is a strict Nash equilibrium and so without loss of generality weights can be assumed to be strictly positive. However, if one wants to extend this proposition to a wider class of games, this condition will be required. ${ }^{23}$

As we noted in Section 4.2 , if $s^{*}$ is a strict $\left(p_{1}, p_{2}\right)$-dominant equilibrium with $p_{1}+p_{2}<$ 1 , then the singleton set $\left\{s^{*}\right\}$ is an LP-max with respect to the finest partition of strategy sets in the case of 2-by-2 games, and with strictly positive weights ([15, Lemma 7$])$. Thus we obtain the following corollary to Proposition 3.

\footnotetext{
${ }^{23}[16]$ define a strict local potential maximizer to be a local potential maximizer with strictly positive weights.
} 
Corollary 1 Suppose that $s^{*}$ is a strict $\left(p_{1}, p_{2}\right)$-dominant equilibrium with $p_{1}+p_{2}<1$. Then it is the unique stochastically stable state under the relative log-linear dynamics, i.e., $\operatorname{Support}\left(\lim _{\beta \rightarrow \infty} \mu^{r}(u, \beta)\right)=\left\{s^{*}\right\}$.

\section{Proof of the Main Theorem}

The proof of Theorem 2 is carried out in a series of steps in which we examine the relationship between the Markov process where players share common payoff function $v$, a local potential function, ( $v$-process), and the Markov process with payoff functions $u=\left(u_{1}, \ldots, u_{i}\right)$ of the given game (u-process). The stochastically stable states of the former process is known from Theorem 1 as it is the log-linear process for a common interest game, hence equivalent to a potential game. They are precisely those strategy profiles that maximize $v$, i.e., the local potential maximizer. In order to show that the local potential maximizer is also stochastically stable under the $u$-process, we compare the transition matrices for the two processes, not only in the limit as $\beta \rightarrow \infty$, but for each $\beta<\infty$.

\subsection{Stochastic Orders}

We say that a set $T_{i} \subset S_{i}$ (resp. $T \subset S$ ) is increasing if $s_{i} \in T_{i}$ and $s_{i} \leq_{i} s_{i}^{\prime}$ imply $s_{i}^{\prime} \in T_{i}$ (resp. $s \in T$ and $s \leq s^{\prime}$ imply $s^{\prime} \in T$ ). Note that a set $T_{i} \in \mathcal{S}_{i}$ is increasing if, and only if

$T_{i}=\varnothing$ or $T_{i}=\cup_{k=j}^{K_{i}} S_{i k}$ for some $0 \leq j \leq K_{i}$. It is easy to see that $T \in \mathcal{S}$ is increasing if, and only if, it is a union of sets of the form $T_{1} \times \cdots \times T_{n}$ where each $T_{i} \in \mathcal{S}_{i}$ is increasing. In particular, if $T \in \mathcal{S}$ is increasing then $T_{i}=\left\{s_{i} \in S_{i} \mid\left(s_{i}, s_{-i}\right) \in T\right.$ for some $\left.s_{-i}\right\}$ belongs to $\mathcal{S}_{i}$ and is increasing for every $i$. For any finite set $X$ we denote the set of probabilities on $X$ by $\Delta(X)$. For any $\mu_{i}, \nu_{i} \in \Delta\left(S_{i}\right)$, we write $\mu_{i} \leq \mathcal{S}_{i} \nu_{i}$ if $\mu_{i}\left(T_{i}\right) \leq \nu_{i}\left(T_{i}\right)$ for any increasing set $T_{i} \in \mathcal{S}_{i}$. Similarly, for any $\mu, \nu \in \Delta(S)$, we write $\mu \leq_{\mathcal{S}} \nu$ if $\mu(T) \leq \nu(T)$ for any increasing set $T \in \mathcal{S}$. The next lemma is standard. We provide a proof for the reader's convenience.

Lemma 1 For any $\mu_{i}, \nu_{i} \in \Delta\left(S_{i}\right), \mu_{i} \leq \mathcal{S}_{i} \nu_{i}$ if, and only if, $\mathrm{E}_{\mu_{i}}[\phi] \leq \mathrm{E}_{\nu_{i}}[\phi]$ for every 
$\mathcal{S}_{i}$-measurable nondecreasing function $\phi: S_{i} \rightarrow \mathbb{R}$, i.e.,

$$
\sum_{s_{i} \in S_{i}} \mu_{i}\left(s_{i}\right) \phi\left(s_{i}\right)=\sum_{k=0}^{K_{i}} \mu_{i}\left(S_{i k}\right) \phi_{k} \leq \sum_{k=0}^{K_{i}} \nu_{i}\left(S_{i k}\right) \phi_{k}=\sum_{s_{i} \in S_{i}} \nu_{i}\left(s_{i}\right) \phi\left(s_{i}\right)
$$

where $\phi\left(s_{i}\right)=\phi_{k}$ whenever $s_{i} \in S_{i k}$, and $\phi_{0} \leq \cdots \leq \phi_{K_{i}}$. Similarly, for any $\mu, \nu \in \Delta(S)$, $\mu \leq_{\mathcal{S}} \nu$ if, and only if, $\mathrm{E}_{\mu}[\phi] \leq \mathrm{E}_{\nu}[\phi]$ for any $\mathcal{S}$-measurable increasing function $\phi: S \rightarrow \mathbb{R}$.

Proof. Suppose that $\mathrm{E}_{\mu_{i}}[\phi] \leq \mathrm{E}_{\nu_{i}}[\phi]$ for every $\mathcal{S}_{i}$-measurable increasing function $\phi$ : $S_{i} \rightarrow \mathbb{R}$. Let $T_{i} \in \mathcal{S}_{i}$ be an increasing set. Then the characteristic function of $T_{i}, \chi_{T_{i}}$, is $\mathcal{S}_{i}$-measurable, nondecreasing, and $\mu_{i}\left(T_{i}\right)=\mathrm{E}_{\mu_{i}}\left[\chi_{T_{i}}\right] \leq \mathrm{E}_{\nu_{i}}\left[\chi_{T_{i}}\right]=\nu_{i}\left(T_{i}\right)$.

Conversely, suppose that $\mu_{i} \leq \mathcal{S}_{i} \nu_{i}$. For any increasing set $T_{i} \in \mathcal{S}_{i}$ we have $\mathrm{E}_{\mu_{i}}\left[\chi_{T_{i}}\right] \leq$ $\mathrm{E}_{\nu_{i}}\left[\chi_{T_{i}}\right]$. Thus for any collection of increasing sets $T_{i}^{1}, \ldots, T_{i}^{m} \in \mathcal{S}_{i}$, any nonnegative constants $a_{1}, \ldots, a_{m}$, and any real number $b$, we have

$$
\mathrm{E}_{\mu_{i}}\left[\sum_{k=1}^{m} a_{k} \chi_{T_{i}^{k}}-b\right] \leq \mathrm{E}_{\nu_{i}}\left[\sum_{k=1}^{m} a_{k} \chi_{T_{i}^{k}}-b\right] .
$$

Any function $\phi: S_{i} \rightarrow \mathbb{R}$ takes finite number of values, say $a_{1}<a_{2}<\cdots<a_{m}$. If $\phi$ is $\mathcal{S}_{i}$-measurable and nondecreasing, then $T_{i}^{k}=\left\{s_{i} \in S_{i} \mid \phi\left(s_{i}\right) \geq a_{k}\right\} \in \mathcal{S}_{i}$ is an increasing set and $S_{i}=T_{i}^{1} \supset T_{i}^{2} \supset \cdots \supset T_{i}^{m}$. Finally, note that $\phi=\sum_{k=1}^{m}\left(a_{k}-a_{k-1}\right) \chi_{T_{i}^{k}}$ where $a_{0}=0$. The proof for $\leq_{\mathcal{S}}$ is similar.

Q.E.D.

Let $P$ and $Q$ be transition matrices of Markov chains on $S$. Denote the " $s$-th row" of $P$ and $Q$ by $p(\cdot \mid s)$ and $q(\cdot \mid s)$, respectively. We write $P \leq_{\mathcal{S}} Q$ if $p(\cdot \mid s) \leq_{\mathcal{S}} q(\cdot \mid s)$ for all $s \in S$.

Lemma 2 If $P \leq_{\mathcal{S}} Q$, then $\mu P \leq_{\mathcal{S}} \mu Q$ for any $\mu \in \Delta(S)$.

Proof. Let $T \in \mathcal{S}$ be an increasing set. Then

$$
(\mu P)(T)=\sum_{s^{\prime} \in T} \sum_{s \in S} \mu_{s} p\left(s^{\prime} \mid s\right)=\sum_{s \in S} \mu_{s} p(T \mid s) \leq \sum_{s \in S} \mu_{s} q(T \mid s)=(\mu Q)(T)
$$

where the inequality follows from $p(\cdot \mid s) \leq \mathcal{S} q(\cdot \mid s)$.

Q.E.D. 


\subsection{Comparison of Markov Chains $Q(u, \beta)$ and $Q(v, \beta)$}

Now suppose that $S_{k_{1}^{*}, \ldots, k_{n}^{*}}$ is a local potential maximizer with respect to $u=\left(u_{1}, \ldots, u_{n}\right)$ with a local potential function $v: S \rightarrow \mathbb{R}$ and $w_{i}(\cdot, \cdot) \equiv 1$ for all $i=1, \ldots, n$. The main idea of the proof is as follows. We split the Markov chain under the local potential function $v, Q(v, \beta)$, into two processes $Q^{-}(v, \beta)$ and $Q^{+}(v, \beta)$ which share the same invariant distribution whose support is contained in the LP-max. The Markov chain under the original payoff functions, $Q(u, \beta)$, is shown to be "sandwiched" between $Q^{-}(v, \beta)$ and $Q^{+}(v, \beta)$ in the stochastic order. Under the additional assumption of supermodularity (of $v$ or each $u_{i}$ ) this order is preserved for the corresponding $t$-step transition matrices. Letting $t$ tend to infinity the invariant distribution for $Q(u, \beta)$ is shown to be similarly sandwiched between the invariant distributions for $Q^{-}(v, \beta)$ and $Q^{+}(v, \beta)$ for every $\beta>0$. The proof is complete by letting $\beta \rightarrow \infty$.

Set $S_{i}^{-}=\underset{k \leq k_{i}^{*}}{\cup} S_{i k}$ and $S^{-}=S_{1}^{-} \times \cdots \times S_{n}^{-}$. Define a modified log-linear choice rule $p_{i}^{-}\left(s_{i}^{\prime} \mid s: f_{i}, \beta\right)$, where $f_{i}=u_{i}$ or $v$, by

$$
p_{i}^{-}\left(s_{i}^{\prime} \mid s: f_{i}, \beta\right)=\mathbf{I}\left(s_{i}^{\prime} \in S_{i}^{-}\right) \frac{p_{i}\left(s_{i}^{\prime} \mid s: f_{i}, \beta\right)}{p_{i}\left(S_{i}^{-} \mid s: f_{i}, \beta\right)}
$$

or, more specifically,

$$
p_{i}^{-}\left(s_{i}^{\prime} \mid s: f_{i}, \beta\right)= \begin{cases}\frac{e^{\beta f_{i}\left(s_{i}^{\prime}, s_{-i}\right)}}{\sum_{s_{i}^{\prime \prime} \in S_{i}^{-}} e^{\beta f_{i}\left(s_{i}^{\prime \prime}, s_{-i}\right)}} & \text { if } s_{i}^{\prime} \in S_{i}^{-} \\ 0 & \text { otherwise. }\end{cases}
$$

Let $Q^{-}(f, \beta)=\left(q_{s s^{\prime}}^{-}(f, \beta)\right)_{s, s^{\prime} \in S}$ where $q_{s s^{\prime}}^{-}(f, \beta)$ is computed by substituting (5.1) into (2.3). Thus $Q^{-}(f, \beta)$ is the transition matrix for a log-linear process where transitions to states outside of $S^{-}$are prohibited.

$$
\begin{aligned}
& \text { Similarly, let } S_{i}^{+}=\underset{k_{i}^{*} \leq k}{\cup} S_{i k}, S^{+}=S_{1}^{+} \times \cdots \times S_{n}^{+} \text {and define } \\
& p_{i}^{+}\left(s_{i}^{\prime} \mid s: f_{i}, \beta\right)=\mathbf{I}\left(s_{i}^{\prime} \in S_{i}^{+}\right) \frac{p_{i}\left(s_{i}^{\prime} \mid s: f_{i}, \beta\right)}{p_{i}\left(S_{i}^{+} \mid s: f_{i}, \beta\right)}= \begin{cases}\frac{e^{\beta f_{i}\left(s_{i}^{\prime}, s_{-i}\right)}}{\sum_{s_{i}^{\prime \prime} \in S_{i}^{+}} e^{\beta f_{i}\left(s_{i}^{\prime \prime}, s_{-i}\right)}} & \text { if } s_{i}^{\prime} \in S_{i}^{+} \\
0 & \text { otherwise. }\end{cases}
\end{aligned}
$$


The corresponding transition probability is denoted by $q_{s s^{\prime}}^{+}(f, \beta)$ and we set $Q^{+}(f, \beta)=$ $\left(q_{s s^{\prime}}^{+}(f, \beta)\right)_{s, s^{\prime} \in S}$.

For each $s \in S$, let $q_{s}(f, \beta)=\left(q_{s s^{\prime}}(f, \beta)\right)_{s^{\prime} \in S}, q_{s}^{-}(f, \beta)=\left(q_{s s^{\prime}}^{-}(f, \beta)\right)_{s^{\prime} \in S}$ and $q_{s}^{+}(f, \beta)=$ $\left(q_{s s^{\prime}}^{+}(f, \beta)\right)_{s^{\prime} \in S}$ be the "s-th row" of $Q(f, \beta), Q^{-}(f, \beta)$ and $Q^{+}(f, \beta)$, respectively. By a slight abuse of notation we denote by $Q(v, \beta)$ etc. when every player has the same payoff function $v$.

Measurability of the local potential function $v$ implies a special structure of the transition matrix $Q^{-}(v, \beta)$ (and $\left.Q^{+}(v, \beta), Q(v, \beta)\right)$. First, if $s$ and $s^{\prime}$ belong to the same measurable rectangle, then $\left(s_{i}^{\prime \prime}, s_{-i}\right)$ and $\left(s_{i}^{\prime \prime}, s_{-i}^{\prime}\right)$ belong to the same measurable rectangle (possibly different from the one containing $s$ and $s^{\prime}$ ). So

$$
\ln \frac{p_{i}^{-}\left(s_{i}^{\prime \prime} \mid s: v, \beta\right)}{p_{i}^{-}\left(s_{i}^{\prime \prime} \mid s^{\prime}: v, \beta\right)}=\beta\left[v\left(s_{i}^{\prime \prime}, s_{-i}\right)-v\left(s_{i}^{\prime \prime}, s_{-i}^{\prime}\right)\right]=0
$$

for any $i$ and $s_{i}^{\prime \prime} \in S_{i}$, and hence

$$
p_{i}^{-}(\cdot \mid s: v, \beta)=p_{i}^{-}\left(\cdot \mid s^{\prime}: v, \beta\right) \quad \text { for all } i
$$

Therefore we have

$$
q_{s}^{-}(v, \beta)=q_{s^{\prime}}^{-}(v, \beta)
$$

whenever $s$ and $s^{\prime}$ belong to the same measurable rectangle.

Proposition $4 Q^{-}(v, \beta) \leq_{\mathcal{S}} Q^{-}(u, \beta) \leq_{\mathcal{S}} Q(u, \beta) \leq_{\mathcal{S}} Q^{+}(u, \beta) \leq_{\mathcal{S}} Q^{+}(v, \beta)$.

Proof. We only show $Q^{-}(v, \beta) \leq_{\mathcal{S}} Q^{-}(u, \beta) \leq_{\mathcal{S}} Q(u, \beta)$ as the proof for $Q(u, \beta) \leq \mathcal{S}$ $Q^{+}(u, \beta) \leq_{\mathcal{S}} Q^{+}(v, \beta)$ is similar.

Let us first verify that $Q^{-}(v, \beta) \leq_{\mathcal{S}} Q^{-}(u, \beta)$, i.e., $q_{s}^{-}(v, \beta) \leq_{\mathcal{S}} q_{s}^{-}(u, \beta)$ for all $s \in S$. Since $q_{s s^{\prime}}^{-}(f, \beta)=\sum_{i=1}^{n} \rho_{i} \mathbf{I}\left(s_{i}^{\prime}=s_{i}\right) p_{i}^{-}\left(s_{i}^{\prime} \mid s: f, \beta\right)$ where $f=v$ or $u$, it suffices to show that $p_{i}^{-}(\cdot \mid s: v, \beta) \leq \mathcal{S}_{i} p_{i}^{-}\left(\cdot \mid s: u_{i}, \beta\right)$ for all $i$ and $s$. Take $S_{i k}, S_{i k^{\prime}} \in \mathcal{S}_{i}$ with $k \leq k^{\prime}$. If $k_{i}^{*}<k^{\prime}$, then by the definition of $p_{i}^{-}$we have

$$
p_{i}^{-}\left(S_{i k} \mid s: v, \beta\right) p_{i}^{-}\left(S_{i k^{\prime}} \mid s: u_{i}, \beta\right)=0=p_{i}^{-}\left(S_{i k} \mid s: v, \beta\right) p_{i}^{-}\left(S_{i k^{\prime}} \mid s: u_{i}, \beta\right)
$$


Suppose that $k^{\prime} \leq k_{i}^{*}$. Then Remark $\left(3^{\prime}-1\right)$ implies that

$$
\frac{e^{\beta v\left(s_{i}^{\prime}, s_{-i}\right)}}{e^{\beta v\left(s_{i}, s_{-i}\right)}} \leq \frac{e^{\beta u_{i}\left(s_{i}^{\prime}, s_{-i}\right)}}{e^{\beta u_{i}\left(s_{i}, s_{-i}\right)}}
$$

for any $s_{i} \in S_{i k}, s_{i}^{\prime} \in S_{i k^{\prime}}$ and $s_{-i} \in S_{-i}$, which in turn implies that

$$
\frac{p_{i}^{-}\left(S_{i k^{\prime}} \mid s: v, \beta\right)}{p_{i}^{-}\left(S_{i k} \mid s: v, \beta\right)}=\frac{\sum_{s_{i}^{\prime} \in S_{i k^{\prime}}} e^{\beta v\left(s_{i}^{\prime}, s_{-i}\right)}}{\sum_{s_{i} \in S_{i k}} e^{\beta v\left(s_{i}, s_{-i}\right)}} \leq \frac{\sum_{s_{i}^{\prime} \in S_{i k^{\prime}}} e^{\beta u_{i}\left(s_{i}^{\prime}, s_{-i}\right)}}{\sum_{s_{i} \in S_{i k}} e^{\beta u_{i}\left(s_{i}, s_{-i}\right)}}=\frac{p_{i}^{-}\left(S_{i k^{\prime}} \mid s: u_{i}, \beta\right)}{p_{i}^{-}\left(S_{i k} \mid s: u_{i}, \beta\right)}
$$

for any $s \in S$. Thus, viewed as probabilities on $\left\{S_{i 1}, \ldots, S_{i K_{i}}\right\}, p_{i}^{-}(\cdot \mid s: v, \beta)$ is smaller than $p_{i}^{-}\left(\cdot \mid s: u_{i}, \beta\right)$ in the likelihood ratio order. Hence, if $T_{i} \in \mathcal{S}_{i}$ is an increasing set, then $p_{i}^{-}\left(T_{i}, \mid s: v, \beta\right) \leq p_{i}^{-}\left(T_{i} \mid s: u_{i}, \beta\right)$ follows by the standard argument.

We now turn to the proof of $Q^{-}(u, \beta) \leq_{\mathcal{S}} Q(u, \beta)$, i.e., $q_{s}^{-}(u, \beta) \leq_{\mathcal{S}} q_{s}(u, \beta)$ for all $s \in S$. Again, it is enough to show that $p_{i}^{-}\left(\cdot \mid s: u_{i}, \beta\right) \leq \mathcal{S}_{i} p_{i}\left(\cdot \mid s: u_{i}, \beta\right)$. Let $T_{i} \in \mathcal{S}_{i}$ be an increasing set. ¿From the definition of $S_{i}^{-} \in \mathcal{S}_{i}$, it is easily seen that either $T_{i} \cap S_{i}^{-}=\varnothing$ or $T_{i} \cup S_{i}^{-}=S_{i}$. If $T_{i} \cap S_{i}^{-}=\varnothing$, then $p_{i}^{-}\left(T_{i} \mid s: u_{i}, \beta\right)=0 \leq p_{i}\left(T_{i} \mid s: u_{i}, \beta\right)$. If $T_{i} \cup S_{i}^{-}=S_{i}$, then $T_{i} \backslash S_{i}^{-}=S_{i} \backslash S_{i}^{-}$. So (omitting reference to $s, u_{i}, \beta$ )

$$
\begin{aligned}
p_{i}\left(T_{i}\right) p_{i}\left(S_{i}^{-}\right) & =\left[p_{i}\left(T_{i} \cap S_{i}^{-}\right)+p_{i}\left(T_{i} \backslash S_{i}^{-}\right)\right] p_{i}\left(S_{i}^{-}\right) \\
& =\left[p_{i}\left(T_{i} \cap S_{i}^{-}\right)+\left(1-p_{i}\left(S_{i}^{-}\right)\right)\right] p_{i}\left(S_{i}^{-}\right) \\
& =\left(1-p_{i}\left(S_{i}^{-}\right)\right) p_{i}\left(S_{i}^{-}\right)+p_{i}\left(S_{i}^{-}\right) p_{i}\left(T_{i} \cap S_{i}^{-}\right) \geq p_{i}\left(T_{i} \cap S_{i}^{-}\right)
\end{aligned}
$$

and hence $p_{i}^{-}\left(T_{i}\right) \leq p_{i}\left(T_{i}\right)$.

Q.E.D.

Lemma 3 Let $T \in \mathcal{S}$ be an increasing set and for each $i=1, \ldots, n$ and $s \in S$ let $T_{i}(s)=\left\{s_{i}^{\prime} \in S_{i} \mid\left(s_{i}^{\prime}, s_{-i}\right) \in T\right\}$. Then,

(a) the functions $\phi_{i}: S \rightarrow \mathbb{R}$ defined by $\phi_{i}(s)=p_{i}^{-}\left(T_{i}(s) \mid s: v, \beta\right)$ and $\phi: S \rightarrow \mathbb{R}$ defined by $\phi(s)=\sum_{s^{\prime} \in T} q_{s s^{\prime}}^{-}(v, \beta)$ are $\mathcal{S}$-measurable, and

(b) if, in addition, the local potential function $v: S \rightarrow \mathbb{R}$ is supermodular, then both $\phi_{i}$ and $\phi$ are nondecreasing. 
Proof. Since

$$
\begin{aligned}
\phi(s)=\sum_{s^{\prime} \in T} q_{s s^{\prime}}^{-}(v, \beta) & =\sum_{s^{\prime} \in T} \sum_{i=1}^{n} \rho_{i} \mathbf{I}\left(s_{-i}^{\prime}=s_{-i}\right) p_{i}^{-}\left(s_{i}^{\prime} \mid s: v, \beta\right) \\
& =\sum_{i=1}^{n} \rho_{i} \sum_{s^{\prime} \in T} \mathbf{I}\left(s_{-i}^{\prime}=s_{-i}\right) p_{i}^{-}\left(s_{i}^{\prime} \mid s: v, \beta\right) \\
& =\sum_{i=1}^{n} \rho_{i} \sum_{s_{i}^{\prime} \in T_{i}(s)} p_{i}^{-}\left(s_{i}^{\prime} \mid s: v, \beta\right)=\sum_{i=1}^{n} \rho_{i} \phi_{i}(s),
\end{aligned}
$$

it is enough to prove the claims only for $\phi_{i}$.

(a) Suppose that $s$ and $s^{\prime}$ belong to the same measurable rectangle. Then $\left(s_{i}^{\prime \prime}, s_{-i}\right)$ and $\left(s_{i}^{\prime \prime}, s_{-i}^{\prime}\right)$ belong to the same measurable rectangle for any $s_{i}^{\prime \prime} \in S_{i}$. Since $T \in \mathcal{S}$, this implies that $T_{i}(s)=T_{i}\left(s^{\prime}\right)$. Hence $\phi_{i}(s)=\phi_{i}\left(s^{\prime}\right)$ by $(5.4)$.

(b) Now suppose that $s \leq s^{\prime}$ and $\bar{s}_{i} \in S_{i \bar{k}}, \hat{s}_{i} \in S_{i \hat{k}}$ with $\bar{k} \leq \hat{k}$. Since $v$ is assumed to be supermodular, we have

$$
v\left(\hat{s}_{i}, s_{-i}\right)-v\left(\bar{s}_{i}, s_{-i}\right) \leq v\left(\hat{s}_{i}, s_{-i}^{\prime}\right)-v\left(\bar{s}_{i}, s_{-i}^{\prime}\right)
$$

and so

which in turn implies that

$$
\frac{e^{\beta v\left(\hat{s}_{i}, s_{-i}\right)}}{e^{\beta v\left(\bar{s}_{i}, s_{-i}\right)}} \leq \frac{e^{\beta v\left(\hat{s}_{i}, s_{-i}^{\prime}\right)}}{e^{\beta v\left(\bar{s}_{i}, s_{-i}^{\prime}\right)}}
$$

$$
\frac{p_{i}^{-}\left(S_{i \hat{k}} \mid s: v, \beta\right)}{p_{i}^{-}\left(S_{i \bar{k}} \mid s: v, \beta\right)}=\frac{\sum_{\hat{s}_{i} \in S_{i \hat{k}}} e^{\beta v\left(\hat{s}_{i}, s_{-i}\right)}}{\sum_{\bar{s}_{i} \in S_{i \bar{k}}} e^{\beta v\left(\bar{s}_{i}, s_{-i}\right)}} \leq \frac{\sum_{\hat{s}_{i} \in S_{i \hat{k}}} e^{\beta v\left(\hat{s}_{i}, s_{-i}^{\prime}\right)}}{\sum_{\bar{s}_{i} \in S_{i \bar{k}}} e^{\beta v\left(\bar{s}_{i}, s_{-i}^{\prime}\right)}}=\frac{p_{i}^{-}\left(S_{i \hat{k}} \mid s^{\prime}: v, \beta\right)}{p_{i}^{-}\left(S_{i \bar{k}} \mid s^{\prime}: v, \beta\right)} .
$$

This shows that, viewed as probabilities on $\left\{S_{i 1}, \ldots, S_{i K_{i}}\right\}, p_{i}^{-}(\cdot \mid s: v, \beta)$ is smaller than $p_{i}^{-}\left(\cdot \mid s^{\prime}: v, \beta\right)$ in the likelihood ratio order. Hence it follows by the standard argument that $p_{i}^{-}\left(T_{i} \mid s: v, \beta\right) \leq p_{i}^{-}\left(T_{i} \mid s^{\prime}: v, \beta\right)$ for any increasing set $T_{i} \in \mathcal{S}_{i}$. Since $T \in \mathcal{S}$ is increasing, it is easy to see that $T_{i}(s) \in \mathcal{S}_{i}$ and $T_{i}(s)$ is increasing for any $s \in S$. In addition, $T_{i}(s) \subset T_{i}\left(s^{\prime}\right)$ whenever $s \leq s^{\prime}$. Hence

$$
\phi_{i}(s)=p_{i}^{-}\left(T_{i}(s) \mid s: v, \beta\right) \leq p_{i}^{-}\left(T_{i}(s) \mid s^{\prime}: v, \beta\right) \leq p_{i}^{-}\left(T_{i}\left(s^{\prime}\right) \mid s^{\prime}: v, \beta\right)=\phi_{i}\left(s^{\prime}\right)
$$

as claimed.

Q.E.D. 
Corollary 2 Suppose that $v$ is supermodular. Then for any $\mu, \nu \in \Delta(S)$ with $\mu \leq_{\mathcal{S}} \nu$, we have $\mu Q^{-}(v, \beta) \leq \mathcal{S} \nu Q^{-}(v, \beta)$ and $\mu Q^{+}(v, \beta) \leq \mathcal{S} \nu Q^{+}(v, \beta)$.

Proof. Let $p=\mu Q^{-}(v, \beta)$ and $q=\nu Q^{-}(v, \beta)$, and let $T \in \mathcal{S}$ be an increasing set. Then

$$
p(T)=\sum_{s^{\prime} \in T} \sum_{s \in S} \mu_{s} q_{s s^{\prime}}^{-}(v, \beta)=\sum_{s \in S} \mu_{s} \sum_{s^{\prime} \in T} q_{s s^{\prime}}^{-}(v, \beta)=\sum_{s \in S} \mu_{s} \phi(s)=\mathrm{E}_{\mu}[\phi]
$$

where $\phi(s)$ is defined as in Lemma 3. Similarly, $q(T)=\mathrm{E}_{\nu}[\phi]$. Since $\mu \leq_{\mathcal{S}} \nu$ and $\phi$ is $\mathcal{S}$-measurable and nondecreasing by Lemma 3 , we have $p(T) \leq q(T)$ by Lemma 1 . Hence $p \leq \mathcal{S} q$. The proof for $Q^{+}(v, \beta)$ is similar and omitted.

Proposition 5 Suppose that either $v$ is supermodular or $u_{i}$ is supermodular for each $i=1, \ldots, n$. Then for any $\mu, \nu \in \Delta(S)$ with $\mu \leq_{\mathcal{S}} \nu$ we have $\mu Q^{-}(v, \beta) \leq_{\mathcal{S}} \nu Q^{-}(u, \beta) \leq \mathcal{S}$ $\nu Q(u, \beta)$ and $\mu Q(u, \beta) \leq \mathcal{S} \mu Q^{+}(u, \beta) \leq \mathcal{S} \nu Q^{+}(v, \beta)$.

Proof. We carry out the proof only for $Q^{-}$. Take $\mu, \nu \in \Delta(S)$ with $\mu \leq_{\mathcal{S}} \nu$ and let $T \in \mathcal{S}$ be an increasing set. Suppose first that $v$ is supermodular. Then $\mu Q^{-}(v, \beta) \leq_{\mathcal{S}} \nu Q^{-}(v, \beta)$ by Corollary 2, and $\nu Q^{-}(v, \beta) \leq_{\mathcal{S}} \nu Q^{-}(u, \beta) \leq_{\mathcal{S}} \nu Q(u, \beta)$ by Proposition 4 and Lemma 2. Hence $\mu Q^{-}(v, \beta) \leq_{\mathcal{S}} \nu Q^{-}(u, \beta) \leq_{\mathcal{S}} \nu Q(u, \beta)$.

Next, suppose that each $u_{i}$ is supermodular. Let $p=\mu Q^{-}(v, \beta), q=\nu Q^{-}(u, \beta)$ and let $T \subset \mathcal{S}$ be an increasing set. For each $s \in S$ let $R(s)$ be the measurable rectangle containing $s$. Define a function $\psi: S \rightarrow \mathbb{R}$ by $\psi(s)=\min _{\hat{s} \in R(s)} \sum_{s^{\prime} \in T} q_{\hat{s} s^{\prime}}^{-}(u, \beta)$. Then (i) $Q^{-}(v, \beta) \leq Q^{-}(u, \beta)$ (Lemma 4) and (5.5) imply that $\phi(s) \leq \psi(s)$ for every $s \in S$ where $\phi$ is defined as in Lemma 3 , and, (ii) by an argument similar to that in the proof of Lemma 
3 it follows that $\psi$ is $\mathcal{S}$-measurable and nondecreasing. Hence,

$$
\begin{array}{rlrl}
p(T)=\sum_{s^{\prime} \in T} \sum_{s \in S} \mu_{s} q_{s s^{\prime}}^{-}(v, \beta) & =\sum_{s \in S} \mu_{s} \sum_{s^{\prime} \in T} q_{s s^{\prime}}^{-}(v, \beta) & \\
& =\sum_{s \in S} \mu_{s} \phi(s) & & \\
& \leq \sum_{s \in S} \mu_{s} \psi(s) & & \text { by (i) } \\
& \leq \sum_{s \in S} \nu_{s} \psi(s) & & \text { by } \mu \leq \mathcal{S} \nu,(\text { ii }), \text { and Lemma } 1 \\
& \leq \sum_{s \in S} \nu_{s} \sum_{s^{\prime} \in T} q_{s s^{\prime}}^{-}(u, \beta) & & \text { by the definition of } \psi \\
& =\sum_{s^{\prime} \in T} \sum_{s \in S} \nu_{s} q_{s s^{\prime}}^{-}(u, \beta)=q(T) &
\end{array}
$$

which shows that $\mu Q^{-}(v, \beta) \leq_{\mathcal{S}} \nu Q^{-}(u, \beta)$. Together with $\nu Q^{-}(u, \beta) \leq_{\mathcal{S}} \nu Q(u, \beta)$ by Lemma 2, the proof is complete.

Q.E.D.

The following Lemma is a straightforward variation of a result by [3].

Lemma 4 (a) $Q^{-}(v, \beta)$ has a unique invariant distribution given by

$$
\mu_{s}^{-}(v, \beta)=\left\{\begin{array}{cl}
\frac{e^{\beta v(s)}}{\sum_{s^{\prime} \in S^{-}} e^{\beta v\left(s^{\prime}\right)}} & \text { if } s \in S^{-} \\
0 & \text { otherwise }
\end{array}\right.
$$

and, similarly, $Q^{+}(v, \beta)$ has a unique invariant distribution given by

$$
\mu_{s}^{+}(v, \beta)=\left\{\begin{array}{cl}
\frac{e^{\beta v(s)}}{\sum_{s^{\prime} \in S^{+}} e^{\beta v\left(s^{\prime}\right)}} & \text { if } s \in S^{+} \\
0 & \text { otherwise }
\end{array}\right.
$$

(b)

$$
\lim _{\beta \rightarrow \infty} \mu_{s}^{-}(v, \beta)=\lim _{\beta \rightarrow \infty} \mu_{s}^{+}(v, \beta)=\left\{\begin{array}{cl}
\frac{1}{\left|S_{k_{1}^{*}, \ldots, k_{n}^{*} \mid}\right|} & \text { if } s \in S_{k_{1}^{*}, \ldots, k_{n}^{*}} \\
0 & \text { otherwise }
\end{array}\right.
$$


Proof. We carry out the proof only for $Q^{-}(v, \beta)$ as the proof for $Q^{+}(v, \beta)$ is similar.

(a) Observe first that $Q^{-}(v, \beta)$ has a unique recurrence class $S^{-}$and it is aperiodic. Hence, $Q^{-}(v, \beta)$ has a unique invariant distribution whose support is $S^{-}$. To see that $\mu^{-}(v, \beta)$ defined by $(5.6)$ is indeed an invariant distribution, hence the unique one, it is enough to check that it satisfies the detailed balance condition, i.e. $\mu_{s}^{-}(v, \beta) q_{s s^{\prime}}^{-}(v, \beta)=$ $\mu_{s^{\prime}}^{-}(v, \beta) q_{s^{\prime} s}^{-}(v, \beta)$ for any $s, s^{\prime} \in S$. This condition is trivially fulfilled if $s$ or $s^{\prime}$ does not belong to $S^{-}$. So assume they do belong to $S^{-}$. The condition is clearly fulfilled if $s=s^{\prime}$, and also if $s$ and $s^{\prime}$ differ in more than one coordinate, since, then, $q_{s s^{\prime}}^{-}(v, \beta)=q_{s^{\prime} s}^{-}(v, \beta)=$ 0 . Now assume that $s$ and $s^{\prime}$ differ exactly in one coordinate, say $i$, that is, $s_{i} \neq s_{i}^{\prime}$ and $s_{-i}=s_{-i}^{\prime}$. Then

$$
\begin{aligned}
\mu_{s}^{-}(v, \beta) q_{s s^{\prime}}^{-}(v, \beta) & =\frac{e^{\beta v(s)}}{\sum_{s^{\prime \prime} \in S^{-}} e^{\beta v\left(s^{\prime \prime}\right)}} \rho_{i} \frac{e^{\beta v\left(s_{i}^{\prime}, s_{-i}\right)}}{\sum_{s_{i}^{\prime \prime} \in S_{i}^{-}} e^{\beta v\left(s_{i}^{\prime \prime}, s_{-i}\right)}} \\
& =\frac{e^{\beta v\left(s^{\prime}\right)}}{\sum_{s^{\prime \prime} \in S^{-}} e^{\beta v\left(s^{\prime \prime}\right)}} \rho_{i} \frac{e^{\beta v\left(s_{i}, s_{-i}^{\prime}\right)}}{\sum_{s_{i}^{\prime \prime} \in S_{i}^{-}} e^{\beta v\left(s_{i}^{\prime \prime}, s_{-i}^{\prime}\right)}}=\mu_{s^{\prime}}^{-}(v, \beta) q_{s^{\prime} s}^{-}(v, \beta)
\end{aligned}
$$

as claimed.

(b) Since $v(s)>v\left(s^{\prime}\right)$ for every $s \in S_{k_{1}^{*}, \ldots, k_{n}^{*}}$ and $s^{\prime} \notin S_{k_{1}^{*}, \ldots, k_{n}^{*}}$, it follows that $\lim _{\beta \rightarrow \infty} \mu_{s}^{-}(v, \beta)>0$ only if $s \in S_{k_{1}^{*}, \ldots, k_{n}^{*}}$. If $s, s^{\prime} \in S_{k_{1}^{*}, \ldots, k_{n}^{*}}$, then $\mathcal{S}$-measurability of $v$ implies that $\mu_{s}^{-}(v, \beta)=\mu_{s^{\prime}}^{-}(v, \beta)$ and so (5.8) follows.

Q.E.D.

We are now ready to complete the proof of Theorem 2. Recall that $Q(u, \beta)$ is irreducible and aperiodic, and its unique invariant distribution is denoted by $\mu(u, \beta)$.

Proof of Theorem 1. It is enough to show that $\mu^{-}(v, \beta) \leq \mathcal{S} \mu(u, \beta) \leq \mathcal{S} \mu^{+}(v, \beta)$. Indeed, these inequalities together with Lemma 4 -(b) imply that the support of $\lim _{\beta \rightarrow \infty} \mu(u, \beta)$ is contained in both $S^{-}$and $S^{+}$, and hence in $S^{-} \cap S^{+}=S_{k_{1}^{*}, \ldots, k_{n}^{*}}$.

By Proposition 4 we have $Q^{-}(v, \beta) \leq_{\mathcal{S}} Q(u, \beta)$. Suppose that $v$ or $u$ is supermodular. Let $\mu, \nu \in \Delta(S)$ be such that $\mu \leq_{\mathcal{S}} \nu$. Then $\mu Q^{-}(v, \beta) \leq_{\mathcal{S}} \nu Q(u, \beta)$ by Proposition 5. Repeated application of Proposition 5 leads to $\mu Q^{-}(v, \beta)^{k} \leq \mathcal{S} \nu Q(u, \beta)^{k}$ for every $k=1,2, \ldots$. Letting $k \rightarrow \infty$ and noting that each row of $\lim _{k \rightarrow \infty} Q^{-}(v, \beta)^{k}$ and 
$\lim _{k \rightarrow \infty} Q(u, \beta)^{k}$ is identical to $\mu^{-}(v, \beta)$ and $\mu(u, \beta)$, respectively, we obtain $\mu^{-}(v, \beta) \leq \mathcal{S}$ $\mu(u, \beta)$. A similar argument shows $\mu(u, \beta) \leq_{\mathcal{S}} \mu^{+}(v, \beta)$.

Q.E.D.

\section{More General Forms of Interaction}

We have so far considered the log-linear process on a strategic form game with a fixed set of players. In this section we consider the log-linear process on a population version of the basic game as well as a model where interactions among players are specified by a finite graph, as in [3]. We will demonstrate that our main results still hold in this set up.

Recall that our basic game is a finite $I$-person game $G$ in strategic form with strategy sets $S_{i}$ and payoff functions $u_{i}: S \rightarrow \mathbb{R}, i=1, \ldots, I$, where $S=S_{1} \times \cdots \times S_{I}$. Each $i=1, \ldots, I$ will now be referred to as a player position.

\subsection{Log-linear Dynamics on a Population Game}

A population game $\Gamma$ associated with $G$ is a finite $I \times N$-person game in strategic form. At each of $I$ player positions there is a population of $N$ players. ${ }^{24}$ Players at the same position $i$ have the identical strategy set, $S_{i}$. An $i$-strategy profile is a profile of strategy choices by $N$ players at position $i$, i.e., a $N$-tuple $\sigma_{i}=\left(s_{i 1}, \ldots, s_{i N}\right) \in \Sigma_{i}=S_{i}^{N}$. A population strategy profile is an $I \times N$-tuple $\sigma=\left(\sigma_{1}, \ldots, \sigma_{I}\right)$ where each $\sigma_{i}$ is an $i$-strategy profile.

Denote by $d_{i}\left(\sigma_{i}\right)=\left(d_{i}\left(s_{i} \mid \sigma_{i}\right)\right)_{s_{i} \in S_{i}}$ the empirical distribution of strategies in $S_{i}$ derived from $\sigma_{i}$, i.e.,

$$
d_{i}\left(s_{i} \mid \sigma_{i}\right)=\frac{n_{i}\left(s_{i} \mid \sigma_{i}\right)}{N}
$$

where $n_{i}\left(s_{i} \mid \sigma_{i}\right)$ is the number of players at position $i$ using strategy $s_{i}$ in $\sigma_{i}$. For each strategy profile $\sigma=\left(\sigma_{1}, \ldots, \sigma_{n}\right)$ let $d(\sigma)=\left(d_{1}\left(\sigma_{1}\right), \ldots, d_{n}\left(\sigma_{n}\right)\right)$. Given a strategy profile $\sigma=\left(\left(s_{11}, \ldots, s_{1 N}\right), \ldots,\left(s_{I 1}, \ldots, s_{I N}\right)\right)$ the payoff to a player $n$ at position $i$ is

$$
U_{i n}(\sigma)=u_{i}\left(s_{i n}, d_{-i}\left(\sigma_{-i}\right)\right) \equiv \sum_{s_{-i} \in S_{-i}} d_{-i}\left(s_{-i} \mid \sigma_{-i}\right) u_{i}\left(s_{i n}, s_{-i}\right)
$$

\footnotetext{
${ }^{24}$ It is only for simplicity of exposition that we assume equal number of players at each position.
} 
where $d_{-i}\left(s_{-i} \mid \sigma_{-i}\right)=\prod_{j \neq i} d_{j}\left(s_{j} \mid \sigma_{j}\right)$. An interpretation is that player $n$ at position $i$ faces $I-1$ players drawn uniformly and independently from each position $j \neq i$, and players at the same position $i$ have the identical payoff function $u_{i}$.

The state space of the dynamic process is the set of (population) strategy profiles, $\Sigma=\Sigma_{1} \times \cdots \times \Sigma_{I}=S_{1}^{N} \times \cdots \times S_{I}^{N}$.

At each period $t \geq 1$ a single player from one, and only one, position is chosen with a positive probability and is given an opportunity to revise her strategy according to the $\log$-linear stochastic choice rule. The probability that a player $n$ at position $i$ receives a revision opportunity is denoted by $\rho_{i n}$ and $\rho_{i n}>0$ for all $i$ and $n$.

If the state in the previous period is $\sigma=\left(\sigma_{1}, \ldots, \sigma_{I}\right)$ where $\sigma_{i}=\left(s_{i 1}, \ldots, s_{i N}\right)$, and if a player $n$ at position $i$ receives a revision opportunity and revises her strategy from $s_{i n}$ to $s_{i n}^{\prime}$, then the new state is $\sigma^{\prime}=\left(\sigma_{i}^{\prime}, \sigma_{-i}\right)$ where $\sigma_{i}^{\prime}=\left(s_{i n}^{\prime}, \sigma_{i(-n)}\right)$. For any two strategy profiles $\sigma$ and $\sigma^{\prime}$ we write $\sigma \stackrel{i, n}{\longrightarrow} \sigma^{\prime}$ if they differ at a single component, i.e., if $\sigma^{\prime}=\left(\left(s_{i n}^{\prime}, \sigma_{i(-n)}\right), \sigma_{-i}\right)$ for some $i, n$ and $s_{i n}^{\prime} \in S_{i}$.

From the way each player's payoff is defined in (6.1) it is clear that the log-linear choice rule depends only on the position of the player but not on the individual name of the player. Thus let $p_{i}\left(s_{i} \mid \sigma: u_{i}, \beta\right)$ be the probability that any player at position $i$ chooses $s_{i} \in S_{i}$ given a state $\sigma \in \Sigma$. As in (2.1) and (2.2),

$$
\ln \frac{p_{i}\left(s_{i}^{\prime} \mid \sigma: u_{i}, \beta\right)}{p_{i}\left(s_{i} \mid \sigma: u_{i}, \beta\right)}=\beta\left(u_{i}\left(s_{i}^{\prime}, d_{-i}\left(\sigma_{-i}\right)\right)-u_{i}\left(s_{i}, d_{-i}\left(\sigma_{-i}\right)\right)\right)
$$

for all $\sigma \in \Sigma$ and $s_{i}, s_{i}^{\prime} \in S_{i}$ or, equivalently,

$$
p_{i}\left(s_{i}^{\prime} \mid \sigma: u_{i}, \beta\right)=\frac{e^{\beta u_{i}\left(s_{i}^{\prime}, d_{-i}\left(\sigma_{-i}\right)\right)}}{\sum_{s_{i} \in S_{i}} e^{\beta u_{i}\left(s_{i}, d_{-i}\left(\sigma_{-i}\right)\right)}} .
$$

The corresponding probability of transition from $\sigma$ to $\sigma^{\prime}$ is given by

$$
q_{\sigma \sigma^{\prime}}(u, \beta)=\sum_{i, n} \mathbf{I}\left(\sigma \stackrel{i, n}{\longrightarrow} \sigma^{\prime}\right) \rho_{i n} p_{i}\left(s_{i n}^{\prime} \mid \sigma: u_{i}, \beta\right) .
$$

Let $Q(u, \beta)$ be the corresponding transition matrix of this Markov chain. Also let $Q^{*}(u)$ be the transition matrix corresponding to the best response choice rule. Note that the log-linear process operates on the population game $\Gamma$, but the transition probabilities and 
matrices are labeled by payoff functions of the underlying game $G$. The population version of the Markov chain is still irreducible and aperiodic. The unique invariant distribution of $Q(u, \beta)$ is again written as $\mu(u, \beta)$. Stochastic stability of a state $\sigma \in \Sigma$ is defined in an identical manner as before.

Potential Games. Suppose that the basic game $G$ is a potential game with a potential function $v$. In the associated population game $\Gamma$, if $\sigma=\left(\left(s_{i k}, \sigma_{i(-k)}\right), \sigma_{-i}\right)$ and $\sigma^{\prime}=$ $\left(\left(s_{i k}^{\prime}, \sigma_{i(-k)}\right), \sigma_{-i}\right)$, then $d_{-i}\left(\sigma_{-i}^{\prime}\right)=d_{-i}\left(\sigma_{-i}\right)$ and

$$
d_{i}\left(s_{i k}^{\prime} \mid \sigma_{i}^{\prime}\right)=\frac{n_{i}\left(s_{i k}^{\prime} \mid \sigma_{i}\right)+1}{N}, \quad d_{i}\left(s_{i k} \mid \sigma_{i}^{\prime}\right)=\frac{n_{i}\left(s_{i k} \mid \sigma_{i}\right)-1}{N} .
$$

It easily follows that

$$
U_{i k}\left(\sigma^{\prime}\right)-U_{i k}(\sigma)=N\left\{v\left(d\left(\sigma^{\prime}\right)\right)-v(d(\sigma))\right\}
$$

where $v(d(\sigma))=\sum_{s \in S} d(s \mid \sigma) v(s)$. That is, $\sigma \mapsto N(v \circ d)(\sigma)$ is a potential function of the population game $\Gamma$.

Clearly, a strategy profile $\sigma$ in $\Gamma$ maximizes the potential function $N(v \circ d)$ if, and only if, the empirical distribution $d(\sigma)$ puts positive probability only on those strategy profiles in $G$ that maximize its potential function $v$, i.e., if, and only if, the support of $d(\sigma)$ is contained in $\operatorname{argmax} v$. Consequently, the set of stochastically stable states can be explicitly characterized simply by restating Theorem 1 .

Corollary 3 (Blume, 1997) Suppose that $G$ is a potential game with a potential function $v$ and hence $\Gamma$ is a potential game with a potential function $N(v \circ d)$. Then the invariant distribution of $Q(v, \beta)$ is

$$
\mu_{\sigma}(v, \beta)=\frac{e^{\beta N v(d(\sigma))}}{\sum_{\sigma \in \Sigma} e^{\beta N v(d(\sigma))}}
$$

and $\sigma \in \Sigma$ is stochastically stable if, and only if, $\operatorname{Support}(d(\sigma)) \subset \operatorname{argmax} v$.

Example 8 Suppose that $G$ is a two-person three-action game with a potential function 


\begin{tabular}{l|l|l|l|}
\multicolumn{1}{c}{} & \multicolumn{1}{c}{0} & \multicolumn{1}{c}{1} & 2 \\
\cline { 2 - 4 } 0 & 1 & 0 & 0 \\
\cline { 2 - 4 } 1 & 0 & 1 & 1 \\
\cline { 2 - 4 } 2 & 0 & 1 & 1 \\
\cline { 2 - 4 } & & &
\end{tabular}

Then the stochastically stable states are $((N, 0,0),(N, 0,0))$ where every player at each position plays strategy 0 , and $((0, N-L, L),(0, N-M, M))$ with $0 \leq L, M \leq N$ where strategies 1 and 2 coexist at each position. When $N=1$, there are five stochastically stable states: $((1,0,0),(1,0,0)),((0,1,0),(0,1,0)),((0,1,0),(0,0,1)),((0,0,1),(0,1,0))$, and $((0,0,1),(0,0,1))$.

Local Potential. From the discussion of the potential games above it is clear that if $S^{*}$ is a local potential maximizer of the underlying game $G$ with a local potential function $v$, then

$$
\Sigma^{*}=\left\{\sigma \in \Sigma \mid \operatorname{Support}(d(\sigma)) \subset S^{*}\right\}
$$

is a local potential maximizer of the population game $\Gamma$ with a local potential function $N(v \circ d) .{ }^{25}$ The next result is thus a restatement of Theorem 2.

Corollary 4 Suppose that $S^{*}$ is a local potential maximizer in $G$ with respect to $u=$ $\left(u_{1}, \ldots, u_{n}\right)$ with a local potential $v: S \rightarrow \mathbb{R}$ and $w_{i}(\cdot, \cdot) \equiv 1$ for all $i=1, \ldots, n$. If $u$ or $v$ is supermodular then the set of stochastically stable states for the log-linear process on $\Gamma$ is contained in $\Sigma^{*}$, i.e., $\operatorname{Support}\left(\lim _{\beta \rightarrow \infty} \mu(u, \beta)\right) \subset \Sigma^{*}$.

The population version of Proposition 2 (when $\beta$ 's differ across player positions or even across individual players) is now obvious and is omitted.

\subsection{The Log-linear Dynamics on Local Interaction Game}

A local interaction over a basic $I$-person game $G$ is described by a multipartite graph $\mathcal{G}=\left(N_{1}, \ldots, N_{I}, E\right)$ where $N_{i} \cap N_{j}=\varnothing$ for any $i$ and $j \neq i$, and $E \subset N \equiv N_{1} \times \cdots \times N_{I}$.

\footnotetext{
${ }^{25}$ The choice of an ordered domain on $\Sigma$ should be obvious.
} 
Each element of $N_{i}$ represents an individual who plays a role of player $i$ in $G$. Each element in $E$ (edges) represents a profile of $I$ players, one from each $N_{i}$, who interact to play $G .{ }^{26}$ We assume that every individual in every $N_{i}$ participates to play $G$ at least once. That is, the projection of $E$ to the $i$-th coordinate is $N_{i}$ itself. For each $i$ and $n \in N_{i}$, let $E_{-i}(n)$ be the collection of $I-1$ players who interact with player $n$. That is, $E_{-i}(n)=\left\{e_{-i} \in N_{-i} \mid\left(n, e_{-i}\right) \in E\right\}$. (As usual, $N_{-i}=\times_{j \neq i} N_{j}$ etc.)

The local interaction game $G(\mathcal{G})$ is an $|N|$-person game where player $n \in N_{i}$ has the strategy set $S_{i}$. Players' choices of strategies are summarized by a configuration $\phi=$ $\left(\phi_{1}, \ldots, \phi_{I}\right)$ where $\phi_{i}: N_{i} \rightarrow S_{i}$ represents choices of strategies by players in $N_{i}$. Given a configuration $\phi$, the payoff to player $n \in N_{i}$ is

$$
U_{n}(\phi)=\sum_{e_{-i} \in E_{-i}(n)} u_{i}\left(\phi_{i}(n), \phi_{-i}\left(e_{-i}\right)\right)
$$

The log-linear dynamics on $G(\mathcal{G})$ is defined just as that on $G$. The state space is now the set of all configurations, $\Phi=S_{1}^{N_{1}} \times \cdots \times S_{I}^{N_{I}}$.

Potential Games. Suppose that $G$ is a potential game. If $v: S \rightarrow \mathbb{R}$ is a potential function for $G$, then it is easy to verify that $V: \Phi \rightarrow \mathbb{R}$ defined by

$$
V(\phi)=\frac{1}{I} \sum_{i=1}^{I} \sum_{n \in N_{i}} \sum_{e_{-i} \in E_{-i}(n)} v\left(\phi_{i}(n), \phi_{-i}\left(e_{-i}\right)\right)
$$

is a potential function for $G(\mathcal{G})$. Clearly, $V$ is maximized at any configuration $\phi=$ $\left(\phi_{1}, \ldots, \phi_{I}\right)$ such that for every edge $e=\left(e_{1}, \ldots, e_{I}\right) \in E$, the strategy profile $\phi(e)=$ $\left(\phi_{1}\left(e_{1}\right), \ldots, \phi_{I}\left(e_{I}\right)\right)$ maximizes $v$. Let $\Phi^{*}$ be the set of such configuration.

\footnotetext{
${ }^{26} \mathrm{In}[3]$ a local interaction over a two-player symmetric game is modeled by a an undirected graph $(N, E)$ where $E \subset N \times N, E$ is disjoint from the diagonal, and every node is connected to at least one other node. The payoff to $n \in N$ at a configuration $\phi: N \rightarrow S$ is given by

$$
g_{n}(\phi)=\sum_{m \in N} \mathbf{1}((n, m) \in E) u(\phi(n), \phi(m))
$$
}

It easy to convert this setup to a bipartite graph $\left(N_{1}, N_{2}, E\right)$. 
Corollary 5 (Blume, 1997) Suppose that $G$ is a potential game with a potential function $v$ and hence $G(\mathcal{G})$ is a potential game with a potential function $V$ defined by (6.7). Then the invariant distribution of the log-linear dynamics on $G(\mathcal{G})$ with parameter $\beta$ is

$$
\mu_{\phi}(V, \beta)=\frac{e^{\beta V(\phi)}}{\sum_{\phi^{\prime} \in \Phi} e^{\beta V\left(\phi^{\prime}\right)}}
$$

and the set of stochastically stable states is precisely $\Phi^{*}$.

Needless to say, precise form of $\Phi^{*}$ depends on the form of interaction, i.e., the graph $\mathcal{G}$. But if there is a unique strategy profile $s^{*}$ that maximizes a potential function $v$ for the basic game $G$, then $\Phi^{*}$ is a singleton set $\left\{\phi^{*}\right\}$ where $\phi^{*}(e)=s^{*}$ for every $e \in E$.

Local Potential. From the discussion of the potential games above it is clear that if $S^{*}$ is a local potential maximizer of the underlying game $G$ with a local potential function $v$, then

$$
\Phi^{*}=\left\{\phi \in \Phi \mid \forall e \in E, \phi(e) \in S^{*}\right\}
$$

is a local potential maximizer of the population game $G(\mathcal{G})$ with a local potential function $V$ defined by (6.7). ${ }^{27}$ The next result is thus a restatement of Theorem 2.

Corollary 6 Suppose that $S^{*}$ is a local potential maximizer in $G$ with respect to $u=$ $\left(u_{1}, \ldots, u_{n}\right)$ with a local potential $v: S \rightarrow \mathbb{R}$ and $w_{i}(\cdot, \cdot) \equiv 1$ for all $i=1, \ldots, n$. If $u$ or $v$ is supermodular then the set of stochastically stable states for the log-linear process on $G(\mathcal{G})$ is contained in $\Phi^{*}$.

Again, the population version of Proposition 2 (when $\beta$ 's differ across player positions or even across individual players) is obvious and is omitted.

\section{Concluding Remarks}

We close with a few remarks. First, in [15, Proposition 3], an LP-max (not necessarily with constant weights) is shown to be robust to incomplete information under an additional

\footnotetext{
${ }^{27}$ Again, the choice of an ordered domain on $\Phi$ should be obvious. Recall that $\Phi=S_{1}^{N_{1}} \times \cdots \times S_{I}^{N_{I}}$.
} 
assumption that either the local potential or the payoff functions exhibit diminishing marginal returns. Under the same additional condition, [16, Lemma 4.5, Corollary 4.6] shows that an LP-max (singleton in their setup) is globally accessible for all sufficiently small degree of friction under the perfect-foresight dynamics. We note that an LP-max with a constant weight as in our theorem would also be robust and globally accessible even if neither $u_{i}$ 's nor $v$ exhibit diminishing marginal returns. Indeed, one can show that an LP-max with a constant weight is a monotone potential maximizer as defined in [15] without diminishing marginal return assumption. A monotone potential maximizer is robust to incomplete information ([15]) as well as linearly absorbing and globally accessible $([16])$.

A simple case of monotone potential maximizer can be described as follows. A strategy profile $s^{*}$ is a monotone potential maximizer if one can find (a) a total order on each player's strategy set with $s_{i}^{*}$ as the maximum and (b) a real valued function $v$ defined on the set of strategy profiles with two conditions. First, $s^{*}$ uniquely maximizes the value of $v$. Second, against any conjecture on other players' strategies, a player has an action that is a best response in the given game and is at least as large in an order found in (a) as some best response action in the common interest game with payoff function $v$. Features of perfect foresight dynamics distinct from the log-linear dynamics are that, first, the relevant solution (stability) concepts are invariant to affine transformation of the underlying game and, second, that it preserves the order of best responses as described above under the two games and, consequently, relevant stability properties of an action under common interest game is inherited by the given game under consideration ([16]). Neither of these properties, appropriately translated, holds under the log-linear dynamics.

Next, it should be noted that our main result (Theorem 2) is not a generalization of Theorem 1 for potential games. While it is true that any potential maximizer is also a local potential maximizer with constant weights in a trivial sense, our result applies if an additional condition of supermodularity is met. Aside from the logical implication of our theorem, this should not be surprising in light of our proof method which indeed relies crucially on the result on potential games.

Finally, we end with a general remark concerning our proof method: comparison of 
Markov chains. ${ }^{28}$ This technique may be useful to characterize the long-run behavior of other dynamic processes. Indeed, if one knows what is the invariant distribution of some given dynamic process on a game with payoffs $u^{\prime}=\left(u_{1},{ }^{\prime}, \ldots, u_{I}^{\prime}\right)$, and if $u=\left(u_{1}, \ldots, u_{I}\right)$ and $u^{\prime}$ are ordered in some appropriate sense - e.g., under the log-linear dynamics and $u^{\prime}=v$ is a local potential function for $u-$ then one could infer information on the invariant distribution for the given dynamics but this time with payoff functions $u$. Thus, our technique seems to be applicable to generalize existing equilibrium selection results in the literature on stochastic (perturbed) best response dynamics (e.g. [18], [10]). While the above statements must remain at an intuitive level for now, a formalization of this general idea is an interesting project for further investigation.

\footnotetext{
${ }^{28}$ See for instance [9], [11].
} 


\section{Appendix}

\section{A Proof of Proposition 1}

Suppose that conditions (a) and (b) of the proposition are satisfied. We will verify that the function $v$ defined by (3.4) is an appropriate local potential function. It is clear from the definition that $v$ is constant on each measurable rectangle and, by (b), its maximum value is attained uniquely on $S^{*}=S_{\boldsymbol{k}^{*}}$ where $\boldsymbol{k}^{*}=\left(k_{1}^{*}, \ldots, k_{I}^{*}\right)$. It remains to show that $v$ satisfies condition (3)-(d) ((d-1) and (d-2)) in Definition 2 with constant weights, $w_{i}(\cdot, \cdot) \equiv 1$ for all $i$. As noted in the discussion following Definition 2, when weights are constant the conditions (d-1) and (d-2) are equivalent to (e-1) and (e-2). So we shall verify the latter conditions. Fix a player $i$ and $s_{-i} \in S_{-i}$. Let $\boldsymbol{k}_{-i} \in \times_{j \neq i}\left\{1, \ldots, K_{j}\right\}$ be the index of the rectangle containing $s_{-i}$, i.e., $s_{-i} \in S_{\boldsymbol{k}_{-i}}$.

To verify (e-1) take $k_{i}<k_{i}^{\prime} \leq k_{i}^{*}, s_{i} \in S_{i k_{i}}$ and $s_{i}^{\prime} \in S_{i k_{i}^{\prime}}$. Let $\boldsymbol{k}=\left(k_{i}, \boldsymbol{k}_{-i}\right)$ and $\boldsymbol{k}^{\prime}=\left(k_{i}^{\prime}, \boldsymbol{k}_{-i}\right)$ so that $\left(s_{i}^{\prime}, s_{-i}\right) \in S_{\boldsymbol{k}}$ and $\left(s_{i}^{\prime}, s_{-i}\right) \in S_{\boldsymbol{k}^{\prime}}$. Let $\boldsymbol{\kappa}^{\prime}=\left(\boldsymbol{k}^{*}, \boldsymbol{k}^{1}, \ldots, \boldsymbol{k}^{\prime}\right)$ be a path that attains the minimum value of $\Lambda$ over $\Pi\left(\boldsymbol{k}^{*}, \boldsymbol{k}^{\prime}\right)$ so that $v\left(s_{i}, s_{-i}\right)=-\Lambda\left(\boldsymbol{\kappa}^{\prime}\right)$. Consider a path $\boldsymbol{\kappa}=\left(\boldsymbol{\kappa}^{\prime}, \boldsymbol{k}\right)\left(\boldsymbol{\kappa}^{\prime}\right.$ followed by $\left.\boldsymbol{k}\right)$. Since $\boldsymbol{\kappa}^{\prime}$ is an individually monotonic path of unilateral deviations terminating at $\boldsymbol{k}^{\prime}=\left(k_{i}^{\prime}, \boldsymbol{k}_{-i}\right)$ with $k_{i}^{\prime} \leq k_{i}^{*}$, it must be that $k_{i}^{*} \geq k_{i}^{1} \geq \cdots \geq k_{i}^{\prime}$. In addition, $k_{i}^{\prime}>k_{i}$ and $\boldsymbol{k}=\left(k_{i}, \boldsymbol{k}_{-i}^{\prime}\right)$, and hence $\boldsymbol{\kappa} \in \Pi\left(\boldsymbol{k}^{*}, \boldsymbol{k}\right)$. By the definition of $v$ we thus have $v\left(s_{i}, s_{-i}\right) \geq-\Lambda(\kappa)$. Therefore,

$$
\begin{aligned}
v\left(s_{i}^{\prime}, s_{-i}\right)-v\left(s_{i}, s_{-i}\right) & \leq \Lambda(\boldsymbol{\kappa})-\Lambda\left(\boldsymbol{\kappa}^{\prime}\right) & & \\
& =\Delta\left(\boldsymbol{k}^{\prime}, \boldsymbol{k}\right) & & \text { by }(3.3) \\
& \leq u_{i}\left(s_{i}^{\prime}, s_{-i}\right)-u_{i}\left(s_{i}, s_{-i}\right) & & \text { by }(3.2)
\end{aligned}
$$

So (e-1) holds. The argument for (e-2) is similar and is omitted.

Conversely, assume that $S^{*}$ is an LP-max with constant weights. Let $\left\{S_{i 1}, \ldots, S_{i K_{i}}\right\}$, $i=1, \ldots, I$, and $v: S \rightarrow \mathbb{R}$ be associated ordered domain and local potential function where $S^{*}=S_{\boldsymbol{k}^{*}}$ with $\boldsymbol{k}^{*}=\left(k_{1}^{*}, \ldots, k_{I}^{*}\right)$. Condition (a) is trivially satisfied by the definition of LP-max. So it suffices to show that (b) holds. We will write the value of $v$ on a measurable rectangle $S_{\boldsymbol{k}}$ by $v_{\boldsymbol{k}}$. Note that $v_{\boldsymbol{k}^{*}}>v_{\boldsymbol{k}}$ for every $\boldsymbol{k} \neq \boldsymbol{k}^{*}$. 
Consider $\boldsymbol{k}^{1}=\left(k_{i}^{1}, \boldsymbol{k}_{-i}^{*}\right)$ where $k_{i}^{1}=k_{i}^{*}-1$ or $k_{i}^{*}+1$. By Definition $2(3)-(\mathrm{d}-1),(\mathrm{d}-2)$ we have

$$
u_{i}\left(s_{i}^{0}, s_{-i}^{0}\right)-u_{i}\left(s_{i}^{1}, s_{-i}^{0}\right) \geq v_{\boldsymbol{k}^{0}}-v_{\boldsymbol{k}^{1}}
$$

for every $s_{i}^{0} \in S_{i k_{i}^{*}}, s_{i}^{1} \in S_{i k_{i}^{1}}$ and $s_{-i}^{*} \in S_{\boldsymbol{k}_{-i}^{*}}$. By taking the minimum over $s_{i}^{0}, s_{i}^{1}$ and $s_{-i}^{0}$ we conclude (recall (3.2))

$$
\Delta\left(\boldsymbol{k}^{*}, \boldsymbol{k}^{1}\right) \geq v_{\boldsymbol{k}^{*}}-v_{\boldsymbol{k}^{1}}
$$

Now, if $\boldsymbol{\kappa}=\left(\boldsymbol{k}^{*}, \boldsymbol{k}^{1}, \ldots, \boldsymbol{k}^{L}\right)$ is an individually monotonic path of unilateral deviations, then by repeating the above argument - noting that $k_{i}^{*} \leq \cdots \leq k_{i}^{L}$ or $k_{i}^{*} \geq \cdots \geq k_{i}^{L}$ for all $i$ - we see that

$$
\Lambda(\boldsymbol{\kappa})=\sum_{\ell=1}^{L} \Delta\left(\boldsymbol{k}^{\ell-1}, \boldsymbol{k}^{\ell}\right) \geq v_{\boldsymbol{k}^{*}}-v_{\boldsymbol{k}^{L}}>0 .
$$

This completes the proof.

Q.E.D.

\section{B p-dominance and Stochastic Stability in $2 \times 2$ Games.}

We show that, for $2 \times 2$ games, a $\left(p_{1}, p_{2}\right)$-dominant equilibrium with $\max \left\{p_{1}, p_{2}\right\}<\frac{1}{2}$ is stochastically stable. The proof uses the tree surgery results for the log-linear dynamics developed by [1] which we explain first. ${ }^{29}$

For each state $s \in S$, a revision $s$-tree is a tree with $S$ as the set of nodes such that from any other state in $S$ there is a unique path of unilateral deviations terminating at $s$. Thus a revision $s$-tree is a tree rooted at $s$ and an edge $\left(s^{\prime}, s^{\prime \prime}\right)$ belongs to it only if $s^{\prime \prime}=\left(s_{i}^{\prime \prime}, s_{-i}^{\prime}\right)$ for some $i$ and $s_{i}^{\prime \prime} \neq s_{i}^{\prime}$. The set of all revision $s$-trees is denoted by $\mathcal{T}(s)$. Define the waste associated with an edge $\left(s^{\prime}, s^{\prime \prime}\right)$ in a revision $s$-tree $T$ by ${ }^{30}$

$$
\mathrm{w}\left(s^{\prime}, s^{\prime \prime}\right)=\max _{\hat{s}_{i} \in S_{i}} u_{i}\left(\hat{s}_{i}, s_{-i}^{\prime}\right)-u_{i}\left(s_{i}^{\prime \prime}, s_{-i}^{\prime}\right)
$$

\footnotetext{
${ }^{29}$ Here we tailor the notations to our setup where only one player revises a strategy at a time. More general form of revision opportunities are considered in [1].

${ }^{30}$ The waste corresponds to the resistance in the language of perturbed Markov chains. In the adaptive learning model of [18] (resp. [10]) the number of mistakes (resp. mutations), i.e. non best response choices of actions, needed to move from one state to another is taken as the resistance.
} 
where $i$ is the player who unilaterally deviates from $s^{\prime}$ to $s^{\prime \prime}$. The waste of a revision $s$-tree $T$ is defined as the sum of the wastes associated with all edges in $T$,

$$
\mathrm{w}(T)=\sum_{\left(s^{\prime}, s^{\prime \prime}\right) \in T} \mathrm{w}\left(s^{\prime}, s^{\prime \prime}\right) .
$$

The stochastic potential of a state $s$ is then defined as

$$
\pi(s)=\min _{T \in \mathcal{T}(s)} \mathrm{w}(T)
$$

A state is stochastically stable if, and only if, it has the smallest stochastic potential among all states.

Theorem 3 ([1]) The set of stochastically stable states for the log-linear dynamics is precisely $\operatorname{argmin} \pi$.

Now consider a $2 \times 2$ game given by the bimatrix below.

\begin{tabular}{c|c|c|}
\multicolumn{1}{c}{0} & \multicolumn{1}{c}{1} \\
\cline { 2 - 3 } 0 & $a_{00}, b_{00}$ & $a_{01}, b_{01}$ \\
\cline { 2 - 3 } 1 & $a_{10}, b_{10}$ & $a_{11}, b_{11}$ \\
\cline { 2 - 3 } & &
\end{tabular}

Suppose that $(1,1)$ is a $\left(p_{1}, p_{2}\right)$-dominant equilibrium, i.e.,

$$
\begin{array}{cc}
p a_{11}+(1-p) a_{10} \geq p a_{01}+(1-p) a_{00} & \text { for all } p \geq p_{1} \\
p b_{11}+(1-p) b_{01} \geq p b_{10}+(1-q) b_{00} & \text { for all } p \geq p_{2} .
\end{array}
$$

where $p_{i}<\frac{1}{2}, i=1,2$. Taking $p=1$ in the two inequalities above we see that $(1,1)$ is an equilibrium. Note that if there is a strict equilibrium different from $(1,1)$, it must be $(0,0)$. If $(0,0)$ is not a strict equilibrium, i.e., $a_{00} \leq a_{10}$ or $b_{00} \leq b_{01}$, then $(1,1)$ is reached with a positive probability from any other state under the best response dynamics. Hence every recurrence class of the best response dynamics includes $(1,1)$ (so there can be only one recurrence class) and hence $(1,1)$ is stochastically stable. ${ }^{31}$

\footnotetext{
${ }^{31}$ Note that the argument so far depends only on $(1,1)$ being an equilibrium.
} 
Suppose that $(0,0)$ is a strict equilibrium, i.e., $a_{00}>a_{10}$ and $b_{00}>b_{01}$. ¿From (B.3) the lower bounds on $p_{1}$ and $p_{2}$ for $(1,1)$ to be $\left(p_{1}, p_{2}\right)$-dominant are

$$
\begin{aligned}
& p_{1}^{*}=\frac{a_{00}-a_{10}}{\left(a_{00}-a_{10}\right)+\left(a_{11}-a_{01}\right)}, \\
& p_{2}^{*}=\frac{b_{00}-b_{01}}{\left(b_{00}-b_{01}\right)+\left(b_{11}-b_{10}\right)} .
\end{aligned}
$$

(Note that $0<p_{i}^{*}<1, i=1,2$.) Therefore, the condition $p_{i}<\frac{1}{2}, i=1,2$, amounts to $a_{00}-a_{10}<a_{11}-a_{01}$ and $b_{00}-b_{01}<b_{11}-b_{10}$. This in particular implies that $a_{01}<a_{11}$ and $b_{10}<b_{11}$ and so $(1,1)$ must be a strict equilibrium. ${ }^{32}$

For stochastic stability it suffices to consider only revision $(0,0)$-trees and $(1,1)$-trees since $(0,0)$ and $(1,1)$ are the only recurrence classes (absorbing states) in the best response dynamics. There are only two revision trees to consider for each of them. See Figure 1. Numbers attached to each arrow is the waste of the edge. From the figure it is clear that $\mathrm{w}(0,0)=\min \left\{a_{11}-a_{01}, b_{11}-b_{10}\right\}$ and $\mathrm{w}(1,1)=\min \left\{a_{00}-a_{10}, b_{00}-b_{01}\right\}$. Hence $\mathrm{w}(1,1)<\mathrm{w}(0,0)$ and so $(1,1)$ is stochastically stable.

The above argument sheds yet more light on the cardinal nature of the log-linear process. Note that $(0,0)$ in the above game is $\left(p_{1}^{* *}, p_{2}^{* *}\right)$-dominant where

$$
p_{1}^{* *}=\frac{a_{11}-a_{01}}{\left(a_{11}-a_{01}\right)+\left(a_{00}-a_{10}\right)}, \quad p_{2}^{* *}=\frac{b_{11}-b_{10}}{\left(b_{11}-b_{10}\right)+\left(b_{00}-b_{01}\right)} .
$$

so that $p_{1}^{*}+p_{1}^{* *}=1$ and $p_{2}^{*}+p_{2}^{* *}=1$. It is easy to see that $p_{1}^{*}+p_{2}^{*}<1$ is equivalent to $\min \left\{p_{1}^{*}, p_{2}^{* *}\right\}<\min \left\{p_{1}^{* *}, p_{2}^{* *}\right\}{ }^{33}$ As we saw in Example 5 (and comments following it) this does not guarantee the stochastic stability of $(1,1)$. In that example,

$$
\begin{gathered}
p_{1}^{* *}=\frac{10}{10+2}=\frac{5}{6}, \quad p_{2}^{* *}=\frac{1}{1+2}=\frac{1}{3}, \\
p_{1}^{*}=\frac{2}{10+2}=\frac{1}{6}, \quad p_{2}^{*}=\frac{2}{1+2}=\frac{2}{3},
\end{gathered}
$$

\footnotetext{
${ }^{32}$ Here we showed that if $(1,1)$ is $\left(p_{1}, p_{2}\right)$-dominant with $p_{1}, p_{2}<1 / 2$ and if $(0,0)$ is a strict equilibrium, then $(1,1)$ must also be strict. In contrast a strict $\mathbf{p}$-dominant equilibrium is always a strict equilibrium for any game.

${ }^{33}$ This is equivalent to $(1,1)$ being risk dominant. See [4] and [18, Section 7].
} 
and $p_{1}^{*}+p_{2}^{*}=\frac{5}{6}<1$ and indeed $\min \left\{p_{1}^{*}, p_{2}^{*}\right\}=\frac{1}{6}<\frac{1}{3}=\min \left\{p_{1}^{* *}, p_{2}^{* *}\right\}$. However, the stochastic potentials of $(0,0)$ and $(1,1)$ are, as we saw from Figure 1 ,

$$
\begin{aligned}
& \mathrm{w}(0,0)=\min \left\{a_{11}-a_{01}, b_{11}-b_{10}\right\}=\min \left\{A p_{1}^{* *}, B p_{2}^{* *}\right\}=1, \\
& \mathrm{w}(1,1)=\min \left\{a_{00}-a_{10}, b_{00}-b_{01}\right\}=\min \left\{A p_{1}^{*}, B p_{2}^{*}\right\}=2 .
\end{aligned}
$$

where $A=\left(a_{11}-a_{01}\right)+\left(a_{00}-a_{10}\right)$ and $B=\left(b_{11}-b_{10}\right)+\left(b_{00}-b_{01}\right)$. Consequently $(0,0)$ is stochastically stable. Thus normalizing constants $(A$ and $B)$ exert an influence on stochastically stable states under the log-linear process. As we showed above, a stronger condition such as $\max \left\{p_{1}, p_{2}\right\}<\frac{1}{2}$ is needed to ensure an equivalence between $\min \left\{p_{1}^{*}, p_{2}^{*}\right\}<\min \left\{p_{1}^{* *}, p_{2}^{* *}\right\}$ (risk dominance) and $\mathrm{w}(1,1)<\mathrm{w}(0,0)$ (stochastic stability). ${ }^{34}$

\footnotetext{
${ }^{34}$ In contrast, [18] shows that the risk dominance suffices for stochastic stability in his version of adaptive learning dynamics.
} 
(a) Revision $(0,0)$-trees
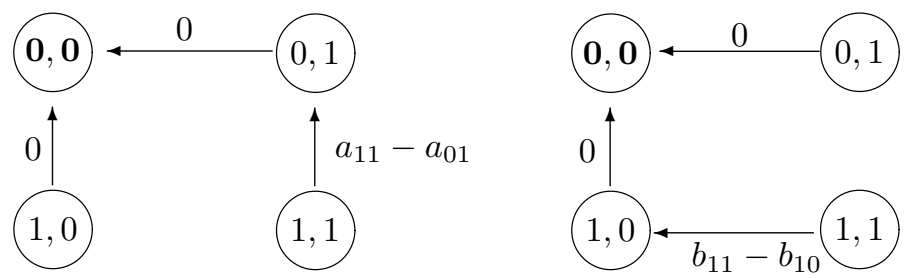

(b) Revision (1,1)-trees
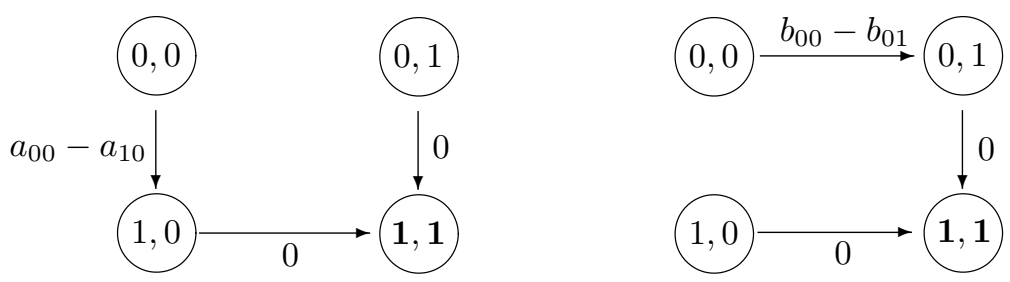

Figure 1: Revision trees for computing stochastic potential 


\section{References}

[1] Carlos Alós-Ferrer and Nick Netzer. The logit-response dynamics, 2008.

[2] Lawrence E. Blume. The statistical mechanics of strategic interaction. Games and Economic Behavior, 5:387-424, 1993.

[3] Lawrence E. Blume. Population games. In B. Arthur et a, editor, The Economy as an Evolving Complex System II, pages 425-460. Addison-Wesley, Reading, MA, 1997.

[4] Hans Carlsson and Eric van Damme. Global games and equilibrium selection. Econometrica, 61(5):989-1018, 1993.

[5] Glenn Ellison. Basins of attraction, long-run stochastic stability, and the speed of step-by-step evolution. The Review of Economic Studies, 67(1):17-45, 2000.

[6] David M. Frankel, Stephen Morris, and Ady Pauzner. Equilibrium selection in global games with strategic complementarities. Journal of Economic Theory, 108:1-44, 2003.

[7] Mark Freidlin and Alexander Wentzell. Random Perturbations of Dynamical Systems. Springer Verlag, New York, 1984.

[8] Atsushi Kajii and Stephen Morris. The robustness of equilibria to incomplete information. Econometrica, 65(6):1283-1309, 1997.

[9] T Kamae, U Urengel, and G. L. O'Brien. Stochastic inequalities on partitially ordered spaces. The Annals of Prpbability, 5(6):899-912, 1977.

[10] Michihiro Kandori, George J. Mailath, and Rafael Rob. Learning, mutation, and long-run equilibria in games. Econometrica, 61:29-56, 11993.

[11] William A. Massey. Stochastic orderings for markov processes on partially ordered spaces. Mathematics of Operations Research, 12(2):350-367, 1987.

[12] Dov Monderer and Lloyd S. Shapley. Potential games. Games and Economic Behavior, 14:124-143, 1996. 
[13] Stephen Morris. Potential methods in interaction games, 1999.

[14] Stephen Morris, Rafael Rob, and Hyung Song Shin. p-dominance and belief potential. Econometrica, 63(1):145-157, 1995.

[15] Stephen Morris and Takashi Ui. Generalized potential and robust sets of equilibria. Journal of Economic Theory, 124:45-78, 2005.

[16] Daisuke Oyama, Satoru Takahashi, and Josef Hofbauer. Monotone methods for equilibrium selection under perfect foresight dynamics. Theoretical Economics, 3(2):155192, 2008.

[17] Takashi Ui. Robust equilibria of potential games. Econometrica, 69:13731380, 2001.

[18] H. Peyton Young. The evolution of conventions. Econometrica, 61(1):57-84, 1993. 